\title{
Radiation and Heat Transfer in the Atmosphere: A Comprehensive Approach on a Molecular Basis
}

\author{
Hermann Harde \\ Laser Engineering and Materials Science, Helmut-Schmidt-University Hamburg, Holstenhofweg 85, 22043 Hamburg, Germany \\ Correspondence should be addressed to Hermann Harde; harde@hsu-hh.de
}

Received 29 April 2013; Accepted 12 July 2013

Academic Editor: Shaocai Yu

Copyright (C) 2013 Hermann Harde. This is an open access article distributed under the Creative Commons Attribution License, which permits unrestricted use, distribution, and reproduction in any medium, provided the original work is properly cited.

\begin{abstract}
We investigate the interaction of infrared active molecules in the atmosphere with their own thermal background radiation as well as with radiation from an external blackbody radiator. We show that the background radiation can be well understood only in terms of the spontaneous emission of the molecules. The radiation and heat transfer processes in the atmosphere are described by rate equations which are solved numerically for typical conditions as found in the troposphere and stratosphere, showing the conversion of heat to radiation and vice versa. Consideration of the interaction processes on a molecular scale allows to develop a comprehensive theoretical concept for the description of the radiation transfer in the atmosphere. A generalized form of the radiation transfer equation is presented, which covers both limiting cases of thin and dense atmospheres and allows a continuous transition from low to high densities, controlled by a density dependent parameter. Simulations of the up- and down-welling radiation and its interaction with the most prominent greenhouse gases water vapour, carbon dioxide, methane, and ozone in the atmosphere are presented. The radiative forcing at doubled $\mathrm{CO}_{2}$ concentration is found to be $30 \%$ smaller than the IPCC-value.
\end{abstract}

\section{Introduction}

Radiation processes in the atmosphere play a major role in the energy and radiation balance of the earth-atmosphere system. Downwelling radiation causes heating of the earth's surface due to direct sunlight absorption and also due to the back radiation from the atmosphere, which is the source term of the so heavily discussed atmospheric greenhouse or atmospheric heating effect. Upward radiation contributes to cooling and ensures that the absorbed energy from the sun and the terrestrial radiation can be rendered back to space and the earth's temperature can be stabilized.

For all these processes, particularly, the interaction of radiation with infrared active molecules is of importance. These molecules strongly absorb terrestrial radiation, emitted from the earth's surface, and they can also be excited by some heat transfer in the atmosphere. The absorbed energy is reradiated uniformly into the full solid angle but to some degree also re-absorbed in the atmosphere, so that the radiation underlies a continuous interaction and modification process over the propagation distance.
Although the basic relations for this interaction of radiation with molecules are already well known since the beginning of the previous century, up to now the correct application of these relations, their importance, and their consequences for the atmospheric system are discussed quite contradictorily in the community of climate sciences.

Therefore, it seems necessary and worthwhile to give a brief review of the main physical relations and to present on this basis a new approach for the description of the radiation transfer in the atmosphere.

In Section 2, we start from Einstein's basic quantumtheoretical considerations of radiation [1] and Planck's radiation law [2] to investigate the interaction of molecules with their own thermal background radiation under the influence of molecular collisions and at thermodynamic equilibrium $[3,4]$. We show that the thermal radiation of a gas can be well understood only in terms of the spontaneous emission of the molecules. This is valid at low pressures with only few molecular collisions as well as at higher pressures and high collision rates. 
In Section 3, also the influence of radiation from an external blackbody radiator and an additional excitation by a heat source is studied. The radiation and heat transfer processes originating from the sun and/or the earth's surface are described by rate equations, which are solved numerically for typical conditions as they exist in the troposphere and stratosphere. These examples right away illustrate the conversion of heat to radiation and vice versa.

In Section 4, we derive the Schwarzschild equation [511] as the fundamental relation for the radiation transfer in the atmosphere. This equation is deduced from pure considerations on a molecular basis, describing the thermal radiation of a gas as spontaneous emission of the molecules. This equation is investigated under conditions of only few intermolecular collisions as found in the upper mesosphere or mesopause as well as at high collision rates as observed in the troposphere. Following some modified considerations of Milne [12], a generalized form of the radiation transfer equation is presented, which covers both limiting cases of thin and dense atmospheres and allows a continuous transition from low to high densities, controlled by a density dependent parameter. This equation is derived for the spectral radiance as well as for the spectral flux density (spectral intensity) as the solid angular integral of the radiance.

In Section 5, the generalized radiation transfer equation is applied to simulate the up- and downwelling radiation and its interaction with the most prominent greenhouse gases water vapour, carbon dioxide, methane, and ozone in the atmosphere. From these calculations, a detailed energy and radiation balance can be derived, reflecting the different contributions of these gases under quite realistic conditions in the atmosphere. In particular, they show the dominant influence of water vapour over the full infrared spectrum, and they explain why a further increase in the $\mathrm{CO}_{2}$ concentration only gives marginal corrections in the radiation budget.

It is not the objective of this paper to explain the fundamentals of the atmospheric greenhouse effect or to prove its existence within this framework. Nevertheless, the basic considerations and derived relations for the molecular interaction with radiation have some direct significance for the understanding and interpretation of this effect, and they give the theoretical background for its general calculation.

\section{Interaction of Molecules with Thermal Bath}

When a gas is in thermodynamic equilibrium with its environment it can be described by an average temperature $T_{G}$. Like any matter at a given temperature, which is in unison with its surrounding, it is also a source of gray or blackbody radiation as part of the environmental thermal bath. At the same time, this gas is interacting with its own radiation, causing some kind of self-excitation of the molecules which finally results in a population of the molecular states, given by Boltzmann's distribution.

Such interaction first considered by Einstein [1] is replicated in the first part of this section with some smaller modifications, but following the main thoughts. In the second part of this section, also collisions between the molecules are

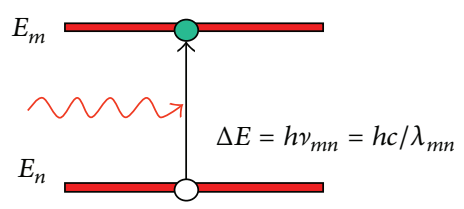

Figure 1: Two-level system with transition between states $m$ and $n$.

included and some basic consequences for the description of the thermal bath are derived.

2.1. Einstein's Derivation of Thermal Radiation. The molecules are characterized by a transition between the energy states $E_{m}$ and $E_{n}$ with the transition energy

$$
\Delta E=E_{m}-E_{n}=h v_{m n}=\frac{h c}{\lambda_{m n}},
$$

where $h$ is Planck's constant, $c$ the vacuum speed of light, $v_{m n}$ the transition frequency, and $\lambda_{m n}$ is the transition wavelength (see Figure 1).

Planckian Radiation. The cavity radiation of a black body at temperature $T_{G}$ can be represented by its spectral energy density $u_{\lambda}$ (units: $\mathrm{J} / \mathrm{m}^{3} / \mu \mathrm{m}$ ), which obeys Planck's radiation law [2] with

$$
u_{\lambda}=\frac{8 \pi n^{4} h c}{\lambda^{5}} \frac{1}{e^{h c / k T_{G} \lambda}-1},
$$

or as a function of frequency assumes the form

$$
u_{v}=u_{\lambda} \frac{\lambda^{2}}{c n}=\frac{8 \pi n^{3} h \nu^{3}}{c^{3}} \frac{1}{e^{h \nu / k T_{G}-1}} .
$$

This distribution is shown in Figure 2 for three different temperatures as a function of wavelength. $n$ is the refractive index of the gas.

Boltzmann's Relation. Due to Boltzmann's principle, the relative population of the states $m$ and $n$ in thermal equilibrium is $[3,4]$

$$
\frac{N_{m}}{N_{n}}=\frac{g_{m}}{g_{n}} e^{-h v_{m n} / k T_{G}},
$$

with $N_{m}$ and $N_{n}$ as the population densities of the upper and lower state, $g_{m}$ and $g_{n}$ the statistical weights representing the degeneracy of these states, $k$ as the Boltzmann constant, and $T_{G}$ as the temperature of the gas.

For the moment, neglecting any collisions of the molecules, between these states three different transitions can take place.

Spontaneous Emission. The spontaneous emission occurs independent of any external field from $m \rightarrow n$ and is characterized by emitting statistically a photon of energy $h v_{m n}$ into the solid angle $4 \pi$ with the probability $d W_{m n}^{s}$ within the time interval $d t$

$$
d W_{m n}^{s}=A_{m n} d t
$$




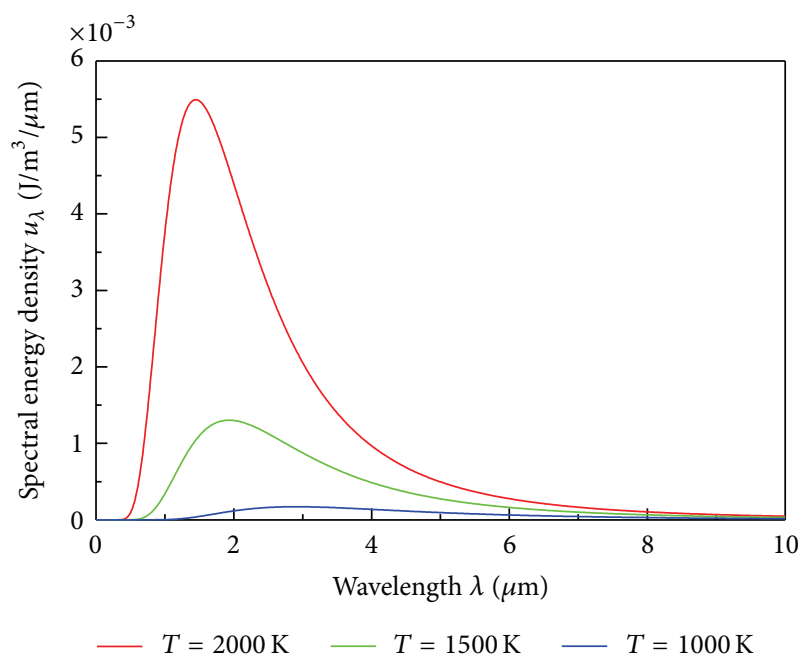

FIGURE 2: Spectral energy density of blackbody radiation at different temperatures.

$A_{m n}$ is the Einstein coefficient of spontaneous emission, sometimes also called the spontaneous emission probability (units: $\mathrm{s}^{-1}$ ).

Induced Absorption. With the molecules subjected to an electromagnetic field, the energy of the molecules can change in that way, that due to a resonant interaction with the radiation the molecules can be excited or de-excited. When a molecule changes from $n \rightarrow m$, it absorbs a photon of energy $h v_{m n}$ and increases its internal energy by this amount, while the radiation energy is decreasing by the same amount.

The probability for this process is found by integrating over all frequency components within the interval $\Delta v$, contributing to an interaction with the molecules:

$$
d W_{n m}^{i}=B_{n m} \int_{\Delta v} u_{\nu} g(\nu) d v \cdot d t
$$

This process is known as induced absorption with $B_{n m}$ as Einstein's coefficient of induced absorption (units: $\mathrm{m}^{3} \cdot \mathrm{Hz} / \mathrm{J} / \mathrm{s}$ ), $u_{v}$ as the spectral energy density of the radiation (units: $\mathrm{J} / \mathrm{m}^{3} / \mathrm{Hz}$ ) and $g(\nu)$ as a normalized lineshape function which describes the frequency dependent interaction of the radiation with the molecules and generally satisfies the relation:

$$
\int_{0}^{\infty} g(v) d v=1
$$

Since $u_{v}$ is much broader than $g(v)$, it can be assumed to be constant over the linewidth, and with (7), the integral in (6) can be replaced by the spectral energy density on the transition frequency:

$$
\int_{\Delta v} u_{\nu} g(\nu) d \nu=u_{\nu_{m n}}
$$

Then the probability for induced absorption processes simply becomes

$$
d W_{n m}^{i}=B_{n m} u_{v_{m n}} d t
$$

Induced Emission. A transition from $m \rightarrow n$ caused by the radiation is called the induced or stimulated emission. The probability for this transition is

$$
d W_{m n}^{i}=B_{m n} \int_{\Delta \nu} u_{\nu} g(\nu) d \nu \cdot d t=B_{m n} u_{\nu_{m n}} d t
$$

with $B_{m n}$ as the Einstein coefficient of induced emission.

Total Transition Rates. Under thermodynamic equilibrium, the total number of absorbing transitions must be the same as the number of emissions. These numbers depend on the population of a state and the probabilities for a transition to the other state. According to (5)-(10), this can be expressed by

$$
B_{n m} u_{v_{m n}} N_{n}=\left(B_{m n} u_{v_{m n}}+A_{m n}\right) N_{m}
$$

or more universally described by rate equations:

$$
\begin{aligned}
& \frac{d N_{m}}{d t}=+B_{n m} u_{v_{m n}} N_{n}-\left(B_{m n} u_{v_{m n}}+A_{m n}\right) N_{m}, \\
& \frac{d N_{n}}{d t}=-B_{n m} u_{v_{m n}} N_{n}+\left(B_{m n} u_{v_{m n}}+A_{m n}\right) N_{m} .
\end{aligned}
$$

For $d N_{m} / d t=d N_{n} / d t=0$, (12) gets identical to (11). Using (4) in (11) gives

$$
g_{n} B_{n m} u_{v_{m n}}=g_{m}\left(B_{m n} u_{v_{m n}}+A_{m n}\right) e^{-h v_{m n} / k T_{G}} .
$$

Assuming that with $T_{G}$ also $u_{v_{m n}}$ gets infinite, $B_{m n}$ and $B_{n m}$ must satisfy the relation:

$$
g_{n} B_{n m}=g_{m} B_{m n} .
$$

Then (13) becomes

$$
B_{m n} u_{v_{m n}} e^{h v_{m n} / k T_{G}}=B_{m n} u_{v_{m n}}+A_{m n}
$$

or resolving to $u_{v_{m n}}$ gives

$$
u_{v_{m n}}=\frac{A_{m n}}{B_{m n}} \frac{1}{e^{h v_{m n} / k T_{G}-1}} .
$$

This expression in Einstein's consideration is of the same type as the Planck distribution for the spectral energy density. Therefore, comparison of (3) and (16) at $\nu_{m n}$ gives for $B_{m n}$ :

$$
B_{m n}=\frac{g_{n}}{g_{m}} B_{n m}=\frac{A_{m n} c^{3}}{8 \pi n^{3} h \nu_{m n}^{3}}=\frac{c}{n} \frac{1}{h \nu_{m n}} \frac{A_{m n} \lambda_{m n}^{2}}{8 \pi n^{2}},
$$

showing that the induced transition probabilities are also proportional to the spontaneous emission rate $A_{m n}$, and in units of the photon energy, $h v_{m n}$, are scaling with $\lambda^{2}$.

2.2. Relationship to Other Spectroscopic Quantities. The Einstein coefficients for induced absorption and emission are directly related to some other well-established quantities in spectroscopy, the cross sections for induced transitions, and the absorption and gain coefficient of a sample. 
2.2.1. Cross Section and Absorption Coefficient. Radiation propagating in $r$-direction through an absorbing sample is attenuated due to the interaction with the molecules. The decay of the spectral energy obeys Lambert-Beer's law, here given in its differential form:

$$
\frac{d u_{v}}{d r}=-\alpha_{n m}(\nu) u_{v}=-\sigma_{n m}(\nu) N_{n} u_{\nu}
$$

where $\alpha_{n m}(\nu)$ is the absorption coefficient (units: $\mathrm{cm}^{-1}$ ) and $\sigma_{n m}(\nu)$ is the cross section (units: $\mathrm{cm}^{2}$ ) for induced absorption.

For a more general analysis, however, also emission processes have to be considered, which partly or completely compensate for the absorption losses. Then two cases have to be distinguished, the situation we discuss in this section, where the molecules are part of an environmental thermal bath, and on the other hand, the case where a directed external radiation prevails upon a gas cloud, which will be considered in the next section.

In the actual case, (18) has to be expanded by two terms representing the induced and also the spontaneous emission. Quite similar to the absorption, the induced emission is given by the cross section of induced emission $\sigma_{m n}(\nu)$, the population of the upper state $N_{m}$, and the spectral radiation density $u_{v}$. The product $\sigma_{m n}(\nu) \cdot N_{m}$ now describes an amplification of $u_{v}$ and is known as gain coefficient.

Additionally, spontaneously emitted photons within a considered volume element and time interval $d t=d r$. $n / c$ contribute to the spectral energy density of the thermal background radiation with

$$
d u_{v}=h v_{m n} A_{m n} d t N_{m} g(v)=\frac{h v_{m n}}{c / n} A_{m n} d r N_{m} g(v) .
$$

Then altogether this gives

$$
\frac{d u_{v}}{d r}=-\sigma_{n m}(\nu) N_{n} u_{v}+\sigma_{m n}(\nu) N_{m} u_{v}+\frac{h v_{m n}}{c / n} A_{m n} N_{m} g(\nu) .
$$

As we will see in Section 2.5 and later in Section 3.3 or Section 4, (19) is the source term of the thermal background radiation in a gas, and (20) already represents the theoretical basis for calculating the radiation transfer of thermal radiation in the atmosphere.

The frequency dependence of $\sigma_{n m}(\nu)$ and $\sigma_{m n}(\nu)$, and thus the resonant interaction of radiation with a molecular transition can explicitly be expressed by the normalized lineshape function $g(v)$ as

$$
\sigma_{m n}(\nu)=\sigma_{m n}^{0} g(\nu), \quad \sigma_{n m}(\nu)=\sigma_{n m}^{0} g(\nu)
$$

Equation (20) may be transformed into the time domain by $d r=c / n \cdot d t$ and additionally integrated over the lineshape $g(\nu)$. When $u_{\nu}$ can be assumed to be broad compared to $g(\nu)$, the energy density $u$ as the integral over the lineshape of width $\Delta v$ becomes

$$
\begin{aligned}
\int_{\Delta v} \frac{d u_{v}}{d t} d v & =\frac{d u_{v_{m n}}}{d t} \Delta v=\frac{d u}{d t} \\
& =-\frac{c}{n}\left(\sigma_{n m}^{0} N_{n}-\sigma_{m n}^{0} N_{m}\right) u_{v_{m n}}+h v_{m n} A_{m n} N_{m}
\end{aligned}
$$

This energy density of the thermal radiation $u$ (units: $\mathrm{J} / \mathrm{m}^{3}$ ) can also be expressed in terms of a photon density $\rho_{G}\left[\mathrm{~m}^{-3}\right]$ in the gas, multiplied with the photon energy $h \cdot v_{m n}$ with

$$
u=u_{v_{m n}} \Delta v=\rho_{G} \cdot h v_{m n}
$$

Since each absorption of a photon reduces the population of state $n$ and increases $m$ by the same amount-for an emission it is just opposite-this yields

$$
\begin{aligned}
\frac{d \rho_{G}}{d t} & =\frac{d N_{n}}{d t}=-\frac{d N_{m}}{d t} \\
& =-\frac{c / n}{h v_{m n}}\left(\sigma_{n m}^{0} N_{n}-\sigma_{m n}^{0} N_{m}\right) u_{v_{m n}}+A_{m n} N_{m}
\end{aligned}
$$

which is identical with the balance in (12). Comparison of the first terms on the right side and applying (17) gives the identity

$$
\begin{aligned}
B_{n m} u_{v_{m n}} N_{n} & =\frac{g_{m}}{g_{n}} \frac{c}{n} \frac{1}{h v_{m n}} \frac{A_{m n} \lambda_{m n}^{2}}{8 \pi n^{2}} u_{v_{m n}} N_{n} \\
& =\frac{c}{n} \frac{1}{h \nu_{m n}} \sigma_{n m}^{0} N_{n} u_{v_{m n}}
\end{aligned}
$$

and therefore

$$
\sigma_{n m}^{0}=\frac{g_{m}}{g_{n}} \frac{A_{m n} \lambda_{m n}^{2}}{8 \pi n^{2}}
$$

Comparing the second terms in (12) and (24) results in

$$
\sigma_{m n}^{0}=\frac{A_{m n} \lambda_{m n}^{2}}{8 \pi n^{2}}=\frac{g_{n}}{g_{m}} \sigma_{n m}^{0}
$$

So, together with (21) and (27), we derive as the final expressions for $\sigma_{n m}(\nu)$ and $\sigma_{m n}(\nu)$ :

$$
\begin{gathered}
\sigma_{m n}(\nu)=\frac{g_{n}}{g_{m}} \sigma_{n m}(\nu)=\frac{A_{m n} \lambda_{m n}^{2}}{8 \pi n^{2}} g(\nu), \\
\sigma_{m n}(\nu)=\frac{h \nu_{m n}}{c / n} B_{m n} g(\nu), \quad \sigma_{n m}(\nu)=\frac{h \nu_{m n}}{c / n} B_{n m} g(\nu) .
\end{gathered}
$$

2.2.2. Effective Cross Section and Spectral Line Intensity. Often the first two terms on the right side of (20) are unified and represented by an effective cross section $\bar{\sigma}_{n m}(\nu)$. Further 


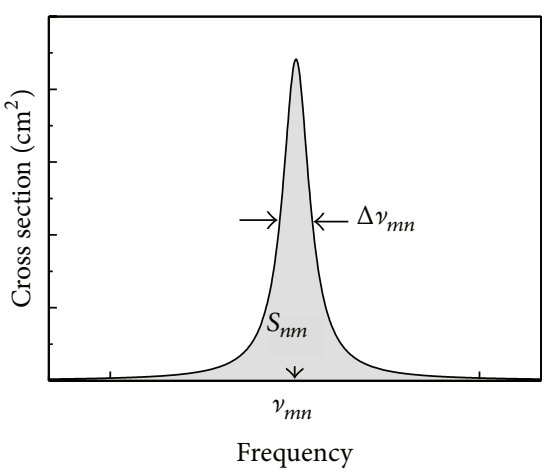

FIgURE 3: For explanation of the spectral line intensity.

relating the interaction to the total number density $N$ of the molecules, it applies

$$
\begin{aligned}
\bar{\sigma}_{n m}(v) N & =\sigma_{n m}(v) N_{n}-\sigma_{m n}(v) N_{m} \\
& =\sigma_{n m}(v) N_{n}\left(1-\frac{g_{n}}{g_{m}} \frac{N_{m}}{N_{n}}\right) \\
& =\frac{h v_{m n}}{c / n} B_{n m} g(v) N_{n}\left(1-\frac{g_{n}}{g_{m}} \frac{N_{m}}{N_{n}}\right),
\end{aligned}
$$

and $\bar{\sigma}_{n m}(\nu)$ becomes

$$
\bar{\sigma}_{n m}(\nu)=\frac{h \nu_{m n}}{c / n} B_{n m} \frac{N_{n}}{N}\left(1-\frac{g_{n}}{g_{m}} \frac{N_{m}}{N_{n}}\right) g(\nu) .
$$

Integration of (30) over the linewidth gives the spectral line intensity $S_{n m}$ of a transition (Figure 3):

$$
S_{n m}=\int_{\Delta v} \bar{\sigma}_{n m}(\nu) d v=\frac{h v_{m n}}{c / n} B_{n m} \frac{N_{n}}{N}\left(1-\frac{g_{n}}{g_{m}} \frac{N_{m}}{N_{n}}\right),
$$

as it is used and tabulated in data bases $[13,14]$ to characterize the absorption strength on a transition.

2.2.3. Effective Absorption Coefficient. Similar to $\bar{\sigma}_{n m}(\nu)$, with (30) and (31), an effective absorption coefficient on a transition can be defined as

$$
\begin{aligned}
\bar{\alpha}_{n m}(\nu) & =\bar{\sigma}_{n m}(\nu) N \\
& =\frac{h \nu_{m n}}{c / n} B_{n m} N_{n}\left(1-\frac{g_{n}}{g_{m}} \frac{N_{m}}{N_{n}}\right) g(\nu) \\
& =S_{n m} N g(\nu)=\bar{\alpha}_{n m}^{0} g(\nu),
\end{aligned}
$$

which after replacing $B_{n m}$ from (17) assumes the more common form:

$$
\begin{aligned}
\bar{\alpha}_{n m}(\nu) & =\frac{A_{m n} c^{2}}{8 \pi n^{2} \nu^{2}} N_{n}\left(\frac{g_{m}}{g_{n}}-\frac{N_{m}}{N_{n}}\right) g(v) \\
& =\frac{A_{m n} \lambda^{2}}{8 \pi n^{2}} N_{n}\left(\frac{g_{m}}{g_{n}}-\frac{N_{m}}{N_{n}}\right) g(\nu) .
\end{aligned}
$$

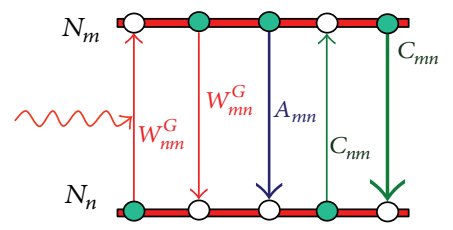

FIGURE 4: Two-level system with transition rates due to stimulated, spontaneous, and collisional processes.

2.3. Collisions. Generally the molecules of a gas underlie collisions, which may perturb the phase of a radiating molecule, and additionally cause transitions between the molecular states. The transition rate from $m \rightarrow n$ due to de-exciting, nonradiating collisions (superelastic collisions, of 2nd type) may be called $C_{m n}$ and that for transitions from $n \rightarrow m$ (inelastic collisions, of 1st type) as exciting collisions $C_{n m}$, respectively (see Figure 4).

2.3.1. Rate Equations. Then, with (25) and the abbreviations $W_{m n}^{G}$ and $W_{n m}^{G}$ as radiation induced transition rates or transition probabilities (units: $\mathrm{s}^{-1}$ )

$$
\begin{aligned}
& W_{m n}^{G}=B_{m n} u_{v_{m n}}=\frac{c}{n} \frac{1}{h v_{m n}} \sigma_{m n}^{0} u_{v_{m n}}, \\
& W_{n m}^{G}=B_{n m} u_{v_{m n}}=\frac{c}{n} \frac{1}{h v_{m n}} \sigma_{n m}^{0} u_{v_{m n}}=\frac{g_{m}}{g_{n}} W_{m n}^{G}
\end{aligned}
$$

the rate equations as generalization of (12) or (24) and additionally supplemented by the balance of the electromagnetic energy density or photon density (see (22)-(24)) assume the form:

$$
\begin{aligned}
& \frac{d N_{m}}{d t}=+\left(W_{n m}^{G}+C_{n m}\right) N_{n}-\left(W_{m n}^{G}+A_{m n}+C_{m n}\right) N_{m} \\
& \frac{d N_{n}}{d t}=-\left(W_{n m}^{G}+C_{n m}\right) N_{n}+\left(W_{m n}^{G}+A_{m n}+C_{m n}\right) N_{m} \\
& \frac{d \rho_{G}}{d t}=-W_{n m}^{G} N_{n}+W_{m n}^{G} N_{m}+A_{m n} N_{m} .
\end{aligned}
$$

At thermodynamic equilibrium, the left sides of (35) are getting zero. Then, also and even particularly in the presence of collisions the populations of states $m$ and $n$ will be determined by statistical thermodynamics. So, adding the first and third equation of (35), together with (4), it is found some quite universal relationship for the collision rates

$$
C_{n m}=\frac{g_{m}}{g_{n}} e^{-h v_{m n} / k T_{G}} C_{m n}
$$

showing that transitions due to inelastic collisions are directly proportional to those of superelastic collisions with a proportionality factor given by Boltzmann's distribution. From (35), it also results that states, which are not connected by an allowed optical transition, nevertheless will assume the same populations as those states with an allowed transition. 
2.3.2. Radiation Induced Transition Rates. When replacing $u_{v_{m n}}$ in (34) by (3), the radiation induced transition rates can be expressed as

$$
W_{m n}^{G}=\frac{A_{m n}}{e^{h v_{m n} / k T_{G}-1}}, \quad W_{n m}^{G}=\frac{g_{m}}{g_{n}} \frac{A_{m n}}{e^{h v_{m n} / k T_{G}-1}} .
$$

Inserting some typical numbers into (37), for example, a transition wavelength of $15 \mu \mathrm{m}$ for the prominent $\mathrm{CO}_{2}$ absorption band and a temperature of $T_{G}=288 \mathrm{~K}$, we calculate a ratio $W_{m n}^{G} / A_{m n}=0.037$. Assuming that $g_{m}=g_{n}$, almost the same is found for the population ratio (see (4)) with $N_{m} / N_{n}=0.036$. At spontaneous transition rates of the order of $A_{m n}=1 \mathrm{~s}^{-1}$ for the stronger lines in this $\mathrm{CO}_{2}$ band then we get a radiation induced transition rate of only $W_{m n}^{G}=W_{n m}^{G}=0.03-0.04 \mathrm{~s}^{-1}$.

Under conditions as found in the troposphere with collision rates between molecules of several $10^{9} \mathrm{~s}^{-1}$, any induced transition rate due to the thermal background radiation is orders of magnitude smaller, and even up to the stratosphere and mesosphere, most of the transitions are caused by collisions, so that above all they determine the population of the states and in any case ensure a fast adjustment of a local thermodynamic equilibrium in the gas.

Nevertheless, the absolute numbers of induced absorption and emission processes per volume, scaling with the population density of the involved states (see (35)), can be quite significant. So, at a $\mathrm{CO}_{2}$ concentration of $400 \mathrm{ppm}$, the population in the lower state $n$ is estimated to be about $N_{n} \sim 7 \times 10^{20} \mathrm{~m}^{-3}$ (dependent on its energy above the ground level). Then more than $10^{19}$ absorption processes per $\mathrm{m}^{3}$ are expected, and since such excitations can take place simultaneously on many independent transitions, this results in a strong overall interaction of the molecules with the thermal bath, which according to Einstein's considerations even in the absence of collisions leads to the thermodynamic equilibrium.

2.4. Linewidth and Lineshape of a Transition. The linewidth of an optical or infrared transition is determined by different effects.

2.4.1. Natural Linewidth and Lorentzian Lineshape. For molecules in rest and without any collisions and also neglecting power broadening due to induced transitions, the spectral width of a line only depends on the natural linewidth

$$
\Delta v_{m n}=\frac{1}{2 \pi \tau_{m}}+\frac{1}{2 \pi \tau_{n}} \approx \frac{A_{m n}}{2 \pi}
$$

which for a two-level system and $\tau_{n} \gg \tau_{m}$ is essentially determined by the spontaneous transition rate $A_{m n} \approx 1 / \tau_{m}$. $\tau_{n}$ and $\tau_{m}$ are the lifetimes of the lower and upper state.

The lineshape is given by a Lorentzian $g_{L}(\nu)$, which in the normalized form can be written as

$$
g_{L}(\nu)=\frac{\Delta v_{m n} / 2 \pi}{\left(\nu-v_{m n}\right)^{2}+\left(\Delta v_{m n} / 2\right)^{2}}, \quad \int_{0}^{\infty} g_{L}(\nu) d \nu=1
$$

2.4.2. Collision Broadening. With collisions in a gas, the linewidth will considerably be broadened due to state and phase changing collisions. Then the width can be approximated by

$$
\Delta v_{m n} \approx \frac{1}{2 \pi}\left(A_{m n}+C_{m n}+C_{n m}+C_{\text {phase }}\right),
$$

where $C_{\text {phase }}$ is an additional rate for phase changing collisions. The lineshape is further represented by a Lorentzian, only with the new homogenous width $\Delta v_{m n}$ (FWHM) according to (40).

2.4.3. Doppler Broadening. Since the molecules are at an average temperature $T_{G}$, they also possess an average kinetic energy

$$
\bar{E}_{\mathrm{kin}}=\frac{3}{2} k T_{G}=\frac{m_{G}}{2} \overline{v^{2}}
$$

with $m_{G}$ as the mass and $\overline{v^{2}}$ as the velocity of the molecules squared and averaged. Due to a Doppler shift of the moving particles, the molecular transition frequency is additionally broadened and the number of molecules interacting within their homogeneous linewidth with the radiation at frequency $v$ is limited. This inhomogeneous broadening is determined by Maxwell's velocity distribution and known as Doppler broadening. The normalized Doppler lineshape is given by a Gaussian function of the form:

$$
g_{D}(\nu)=\frac{2 \sqrt{\ln 2}}{\sqrt{\pi} \Delta \nu_{D}} \exp \left(-\frac{\left(\nu-v_{m n}\right)^{2}}{\left(\Delta \nu_{D} / 2\right)^{2}} \ln 2\right)
$$

with the Doppler linewidth

$$
\Delta \nu_{D}=2 v_{m n}\left(\frac{2 k T_{G}}{m_{G} c^{2}} \ln 2\right)^{1 / 2}=7.16 \cdot 10^{-7} v_{m n} \sqrt{\frac{T_{G}}{M}}
$$

where $M$ is the molecular weight in atomic units and $T_{G}$ specified in $\mathrm{K}$.

2.4.4. Voigt Profile. For the general case of collision and Doppler broadening, a convolution of $g_{D}\left(\nu^{\prime}\right)$ and $g_{L}(\nu-$ $\nu^{\prime}$ ) gives the universal lineshape $g_{V}(\nu)$ representing a Voigt profile of the form:

$$
\begin{aligned}
g_{V}(v)= & \int_{0}^{\infty} g_{D}\left(v^{\prime}\right) g_{L}\left(v-v^{\prime}\right) d v^{\prime} \\
= & \frac{\sqrt{\ln 2}}{\pi \sqrt{\pi}} \frac{\Delta v_{m n}}{\Delta v_{D}} \\
& \times \int_{0}^{\infty} \exp \left(-\frac{\left(v^{\prime}-v_{m n}\right)^{2}}{\left(\Delta v_{D} / 2\right)^{2}} \ln 2\right) \\
& \cdot \frac{d v^{\prime}}{\left(\nu-v^{\prime}\right)^{2}+\left(\Delta v_{m n} / 2\right)^{2}} .
\end{aligned}
$$


2.5. Spontaneous Emission as Thermal Background Radiation. From the rate equations (35), it is clear that also in the presence of collisions, the absolute number of spontaneously emitted photons per time should be the same as that without collisions. This is also a consequence of (36) indicating that with an increasing rate $C_{m n}$ for de-exciting transitions (without radiation), also the rate $C_{n m}$ of exiting collisions is growing and just compensating for any losses, even when the branching ratio $R=A_{m n} / C_{m n}$ of radiating to nonradiating transitions decreases. In other words, when the molecule is in state $m$, the probability for an individual spontaneous emission act is reduced by the ratio $R$, but at the same time, the number of occurring decays per time increases with $C_{m n}$.

Then the spectral power density in the gas due to spontaneous emissions is (see (19))

$$
\frac{d u_{v}}{d t}=h v_{m n} A_{m n} N_{m} g(v)
$$

representing a spectral generation rate of photons of energy $h v_{m n}$ per volume. Photons emerging from a volume element generally spread out into neighbouring areas, but in the same way, there is a backflow from the neighbourhood, which in a homogeneous medium just compensates these losses. Nevertheless, photons have an average lifetime, before they are annihilated due to an absorption in the gas. With an average photon lifetime

$$
\tau_{\mathrm{ph}}=l_{\mathrm{ph}} \frac{n}{c}=\frac{n}{\bar{\alpha}_{n m} c}
$$

where $l_{\mathrm{ph}}=1 / \bar{\alpha}_{n m}$ is the mean free path of a photon in the gas before it is absorbed, we can write for the spectral energy density:

$$
u_{v}=h v_{m n} A_{m n} \tau_{\mathrm{ph}} N_{m} g(\nu)=\frac{n}{c} h v_{m n} \frac{A_{m n}}{\bar{\alpha}_{n m}} N_{m} g(\nu) .
$$

The same result is derived when transforming (20) into the time domain and assuming a local thermodynamic equilibrium with $d u_{v} / d t=0$ :

$$
\begin{aligned}
\left(\sigma_{n m}(\nu) N_{n}-\sigma_{m n}(\nu) N_{m}\right) u_{v} & =\bar{\alpha}_{n m} u_{\nu} \\
& =\frac{n}{c} h v_{m n} A_{m n} N_{m} g(\nu)
\end{aligned}
$$

With $\bar{\alpha}_{n m}(\nu)$ from (33) and Boltzmann's relation (4), then the spectral energy density at $\nu_{m n}$ is found to be

$$
\begin{aligned}
u_{v_{m n}} & =\frac{8 \pi n^{3} h v_{m n}^{3}}{c^{3}} \frac{1}{\left(g_{m} N_{n} / g_{n} N_{m}-1\right)} \\
& =\frac{8 \pi n^{3} h v_{m n}^{3}}{c^{3}} \frac{1}{e^{h v_{m n} / k T_{G}-1}} .
\end{aligned}
$$

This is the well-known Planck formula (3) and shows that without an additional external excitation at thermodynamic equilibrium, the spontaneous emission of molecules can be understood as nothing else as the thermal radiation of a gas on the transition frequency.
This derivation differs insofar from Einstein's consideration leading to (16), as he concluded that a radiation field, interacting with the molecules at thermal and radiation equilibrium, just had to be of the type of a Planckian radiator, while here we consider the origin of the thermal radiation in a gas sample, which exclusively is determined and rightfully defined by the spontaneous emission of the molecules themselves. This is also valid in the presence of molecular collisions. Because of this origin, the thermal background radiation only exists on discrete frequencies, determined by the transition frequencies and the linewidths of the molecules, as long as no external radiation is present. But on these frequencies, the radiation strength is the same as that of a blackbody radiator.

Since this spontaneous radiation is isotropically emitting photons into the full solid angle $4 \pi$, in average half of the radiation is directed upward and half downward.

\section{Interaction of Molecules with Thermal Radiation from an External Source}

In this section, we consider the interaction of molecules with an additional blackbody radiation emitted by an external source like the earth's surface or adjacent atmospheric layers. We also investigate the transfer of absorbed radiation to heat in the presence of molecular collisions, causing a rise of the atmospheric temperature. And vice versa, we study the transfer of a heat flux to radiation resulting in a cooling of the gas. An appropriate means to describe the mutual interaction of these processes is to express this by coupled rate equations which are solved numerically.

\subsection{Basic Quantities}

3.1.1. Spectral Radiance. The power radiated by a surface element $d A$ on the frequency $v$ in the frequency interval $d v$ and into the solid angle element $d \Omega$ is also determined by Planck's radiation law:

$$
\begin{aligned}
& I_{\nu, \Omega}\left(T_{E}\right) d A \cos \beta d \nu d \Omega \\
& \quad=\frac{2 h \nu^{3} n^{2}}{c^{2}} \frac{1}{e^{h \nu / k T_{E}}-1} d A \cos \beta d \nu d \Omega,
\end{aligned}
$$

with $I_{\nu, \Omega}\left(T_{E}\right)$ as the spectral radiance (units: $\mathrm{W} / \mathrm{m}^{2} /$ $\mathrm{Hz} /$ sterad) and $T_{E}$ as the temperature of the emitting surface of the source (e.g., earth's surface). The cosine term accounts for the fact that for an emission in a direction given by the azimuthal angle $\varphi$ and the polar angle $\beta$, only the projection of $d A$ perpendicular to this direction is efficient as radiating surface (Lambertian radiator).

3.1.2. Spectral Flux Density-Spectral Intensity. Integration over the solid angle $\Omega$ gives the spectral flux density. Then, representing $d \Omega$ in spherical coordinates as $d \Omega=\sin \beta d \beta d \varphi$ 


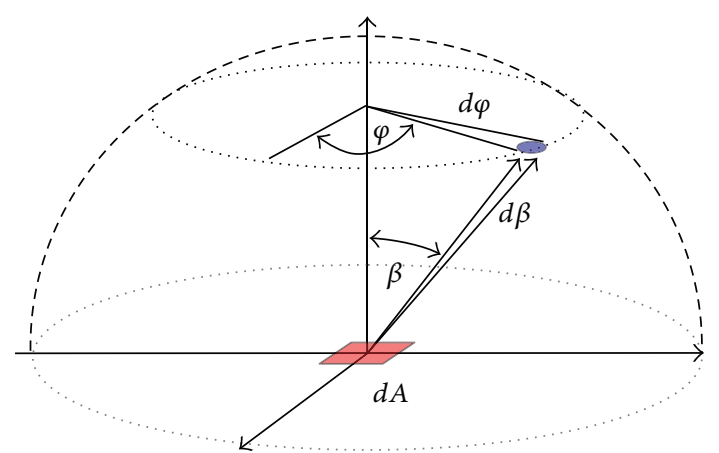

Figure 5: Radiation from a surface element $d A$ into the solid angle $d \Omega=\sin \beta d \beta d \varphi$.

with $d \varphi$ as the azimuthal angle interval and $d \beta$ as the polar interval (see Figure 5), this results in:

$$
\begin{aligned}
I_{\nu} & =\int_{\Omega}^{2 \pi} I_{\nu, \Omega}\left(T_{E}\right) \cos \beta d \Omega \\
& =\int_{\varphi=0}^{2 \pi} \int_{\beta=0}^{\pi / 2} I_{\nu, \Omega}\left(T_{E}\right) \cos \beta \sin \beta d \beta d \varphi \\
& =\frac{2 \pi h \nu^{3} n^{2}}{c^{2}} \frac{1}{e^{h v / k T_{E}}-1}
\end{aligned}
$$

or in wavelengths units

$$
I_{\lambda}=\frac{c n}{\lambda^{2}} I_{\nu}=\frac{2 \pi h c^{2} n^{3}}{\lambda^{5}} \frac{1}{e^{h c / k T_{E} \lambda}-1}
$$

$I_{\lambda}$ and $I_{\nu}$, also known as spectral intensities, are specified in units of $\mathrm{W} / \mathrm{m}^{2} / \mu \mathrm{m}$ and $\mathrm{W} / \mathrm{m}^{2} / \mathrm{Hz}$, respectively. They represent the power flux per frequency or wavelength and per surface area into that hemisphere, which can be seen from the radiating surface element. The spectral distribution of a Planck radiator of $T=299 \mathrm{~K}$ as a function of wavelength is shown in Figure 6.

\subsection{Ray Propagation in a Lossy Medium}

3.2.1. Spectral Radiance. Radiation passing an absorbing sample generally obeys Lambert-Beer's law, as already applied in (18) for the spectral energy density $u_{v}$. The same holds for the spectral radiance with

$$
\frac{d I_{\nu, \Omega}}{d r}=-\bar{\alpha}_{n m}(v) I_{v, \Omega}
$$

where $d r$ is the propagation distance of $I_{\nu, \Omega}$ in the sample. This is valid independent of the chosen coordinate system. The letter $r$ is used when not explicitly a propagation

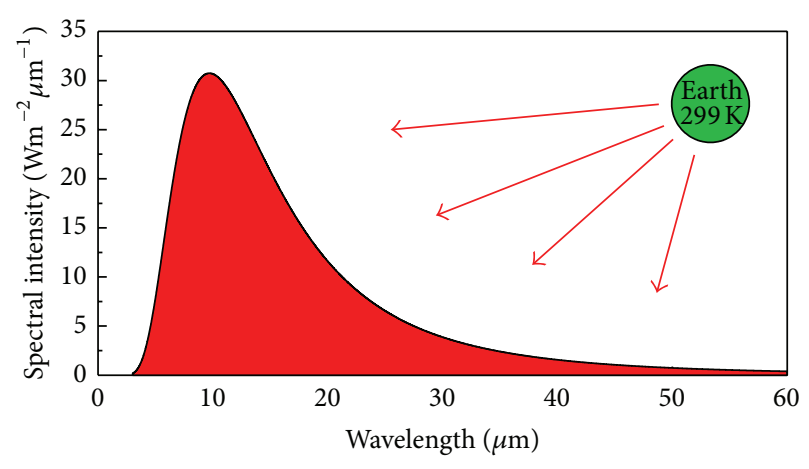

FIGURE 6: Spectrum of a Planck radiator at $299 \mathrm{~K}$ temperature.

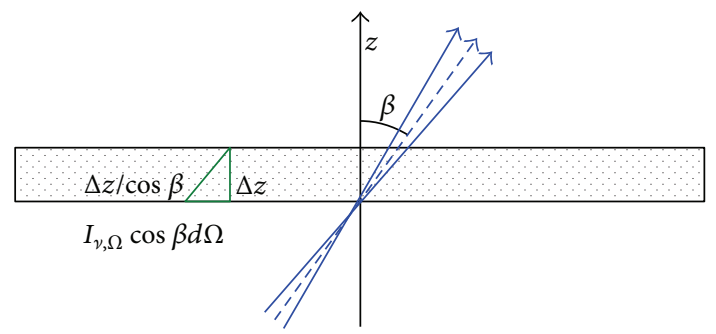

FIGURE 7: Radiation passing a gas layer.

perpendicular to the earth's surface or a layer ( $z$-direction) is meant.

3.2.2. Spectral Intensity. For the spectral intensity, which due to the properties of a Lambertian radiator consists of a bunch of rays with different propagation directions and brightness, some basic deviations have to be recognized. So, because of the individual propagation directions spreading over a solid angle of $2 \pi$, only for a homogeneously absorbing sphere and in a spherical coordinate system with the radiation source in the center of this sphere the distances to pass the sample, and thus the individual contributions to the overall absorption, would be the same.

But radiation, emerging from a plane parallel surface and passing an absorbing layer of thickness $\Delta z$, is better characterized by its average expansion perpendicular to the layer surface in $z$-direction. This means, that with respect to this direction an individual ray, propagating under an angle $\beta$ to the surface normal covers a distance $\Delta l=\Delta z / \cos \beta$ before leaving the layer (see Figure 7). Therefore, such a ray on one side contributes to a larger relative absorption, and on the other side, it donates this to the spectral intensity only with weight $I_{\gamma, \Omega} \cos \beta d \Omega$ due to Lambert's law.

This means that to first order, each individual beam direction suffers from the same absolute attenuation, and particularly the weaker rays under larger propagation angles $\beta$ waste relatively more of their previous spectral radiance. Thus, especially at higher absorption strengths and longer propagation lengths, the initial Lambertian distribution will be more and more modified. A criterion for an almost unaltered distribution may be that for angles $\beta \leq \beta_{\max }$ the inequality $\bar{\alpha}_{n m} \cdot \Delta z / \cos \beta \ll 1$ is satisfied. The absorption 
loss for the spectral intensity as the integral of the spectral radiance $I_{\gamma, \Omega}$ (see (51)) then is given by

$$
\begin{aligned}
& \frac{\int d I_{\nu, \Omega}(z) \cos \beta d \Omega}{d z} \\
& \quad=-\bar{\alpha}_{n m}(\nu) \int_{0}^{2 \pi} \int_{0}^{\pi / 2} I_{\nu, \Omega} \frac{\cos \beta}{\cos \beta} \sin \beta d \beta d \varphi .
\end{aligned}
$$

The cosine terms under the integral sign just compensate and (54) can be written as

$$
\frac{d I_{\nu}\left(z, T_{E}\right)}{d z}=-2 \bar{\alpha}_{n m}(\nu) I_{\nu}\left(z, T_{E}\right) .
$$

This differential equation for the spectral intensity shows that the effective absorption coefficient is twice that of the spectral radiance, or in other words, the average propagation length of the radiation to pass the layer is twice the layer thickness. This last statement means that we also can assume radiation, which is absorbed at the regular absorption coefficient $\bar{\alpha}_{n m}$, but is propagating as a beam under an angle of $60^{\circ}$ to the surface normal $\left(1 / \cos 60^{\circ}=2\right)$. In practice, it even might give sense to deviate from an angle of $60^{\circ}$, to compensate for deviations of the earth's or oceanic surface from a Lambertian radiator, and to account for contributions due to Mie and Rayleigh scattering.

The absorbed spectral power density $\bar{L}_{v}$ (power per volume and per frequency) with respect to the $z$-direction then is found to be

$$
\bar{L}_{\nu}(z)=\frac{d I_{\nu}(z)}{d z}=-2 \bar{\alpha}_{n m}(v) I_{\nu}(0) e^{-2 \bar{\alpha}_{n m}(v) z},
$$

with $I_{\nu}(0)$ as the initial spectral intensity at $z=0$ as given by (51).

An additional decrease of $\bar{L}_{v}$ with $z$ due to a lateral expansion of the radiation over the hemisphere can be neglected, since any propagation of the radiation is assumed to be small compared to the earth's radius (consideration of an extended radiating parallel plane).

Integration of (56) over the lineshape $g(v)$ within the spectral interval $\Delta \nu$ gives the absorbed power density $\bar{L}$ (units: $\mathrm{W} / \mathrm{m}^{3}$ ), which similar to (23) may also be expressed as loss or annihilation of photons per volume $\left(\rho_{E}\right.$ in $\left.\mathrm{m}^{-3}\right)$ and per time. Since the average propagation speed of $I_{\nu}$ in $z$-direction is $c / 2 n$, also the differentials transform as $d z=c / 2 n \cdot d t$. With $I_{\nu}$ from (51), this results in

$$
\begin{aligned}
\bar{L}(z) & =\frac{2 n}{c} \int_{\Delta v} \frac{d I_{v}}{d t} d v=\frac{2 n}{c} \frac{d I_{\nu_{m n}}}{d t} \Delta v=2 \frac{d \rho_{E}}{d t} h v_{m n} \\
& =-\frac{A_{m n}}{2} N_{n}\left(\frac{g_{m}}{g_{n}}-\frac{N_{m}}{N_{n}}\right) \frac{h v_{m n}}{e^{h v_{m n} / k T_{E}}-1} e^{-2 \overline{\bar{\alpha}}_{n m} z},
\end{aligned}
$$

or for the photon density $\rho_{E}$ in terms of the induced transition rates $W_{n m}^{E}$ and $W_{m n}^{E}$ caused by the external field:

$$
\begin{aligned}
\frac{d \rho_{E}}{d t} & =-W_{n m}^{E} N_{n}+W_{m n}^{E} N_{m} \\
& =-\frac{A_{m n}}{4}\left(\frac{g_{m}}{g_{n}} N_{n}-N_{m}\right) \frac{1}{e^{h \nu_{m n} / k T_{E}}-1} e^{-2 \overline{\bar{\alpha}}_{n m} z},
\end{aligned}
$$

with $\overline{\bar{\alpha}}_{n m}=\bar{\alpha}_{n m}^{0} / \Delta v=S_{n m} N / \Delta v$ as an averaged absorption coefficient over the linewidth (see also (32)) and with

$$
\begin{gathered}
W_{m n}^{E}(z)=\frac{A_{m n} \lambda_{m n}^{2}}{8 \pi n^{2} h v_{m n}} I_{\nu_{m n}}(z)=\frac{\sigma_{m n}^{0}}{h \nu_{m n}} I_{\nu_{m n}}(z) \\
=\frac{A_{m n}}{4} \frac{e^{-2 S_{n m} N z / \Delta v}}{e^{h \nu_{m n} / k T_{E}}-1}, \\
W_{n m}^{E}=\frac{g_{m}}{g_{n}} W_{m n}^{E} .
\end{gathered}
$$

Similar to (37), these rates are again proportional to the spontaneous transition probability (rate) $A_{m n}$, but now they depend on the temperature $T_{E}$ of the external source and the propagation depth $z$.

Due to the fact that radiation from a surface with a Lambertian distribution and only from one hemisphere is acting on the molecules, different to (37), a factor of $1 / 4$ appears in this equation.

At a temperature $T_{E}=288 \mathrm{~K}$ and wavelength $\lambda=15 \mu \mathrm{m}$, for example, the ratio of induced to spontaneous transitions due to the external field at $z=0$ is

$$
\frac{W_{m n}^{E}}{A_{m n}}=0.0093 \approx 1 \% .
$$

For the case of a two-level system, $A_{m n}$ can also be expressed by the natural linewidth of the transition with (see (38))

$$
\Delta \nu_{m n}^{N}=\frac{A_{m n}}{2 \pi} .
$$

Then (59) becomes

$$
W_{m n}^{E}(z)=\frac{\pi}{2} \Delta v_{m n}^{N} \frac{e^{-2 S_{n m} N z / \Delta v}}{e^{h v_{m n} / k T_{E}}-1} .
$$

With a typical natural width of the order of only $0.1 \mathrm{~Hz}$ in the $15 \mu \mathrm{m}$ band of $\mathrm{CO}_{2}$, then the induced transition rate will be less than $0.01 \mathrm{~s}^{-1}$. Even on the strongest transitions around $4.2 \mu \mathrm{m}$, the natural linewidths are only $100 \mathrm{~Hz}$, and therefore the induced transition rates are about $10 \mathrm{~s}^{-1}$.

Despite of these small rates, the overall absorption of an incident beam can be quite significant. In the atmosphere, the greenhouse gases are absorbing on hundred thousands of transitions over long propagation lengths and at molecular number densities of $10^{19}-10^{23}$ per $\mathrm{m}^{3}$. On the strongest lines in the $15 \mu \mathrm{m}$ band of $\mathrm{CO}_{2}$, the absorption coefficient at the center of a line even goes up to $1 \mathrm{~m}^{-1}$. Then already within a distance of a few $\mathrm{m}$, the total power will be absorbed on these frequencies. So, altogether about $85 \%$ of the total IR radiation emerging from the earth's surface will be absorbed by these gases.

3.2.3. Alternative Calculation for the Spectral Intensity. For some applications, it might be more advantageous, first to solve the differential equation (53) for the spectral radiance as a function of $z$ and also $\beta$, before integrating over $\Omega$. 


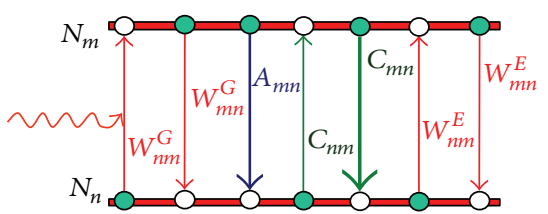

Figure 8: Two-level system with transition rates including an external excitation.

Then an integration in $z$-direction over the length $z / \cos \beta$ with a $z$-dependent absorption coefficient gives

$$
I_{\nu, \Omega}(z)=I_{\nu, \Omega}(0) e^{-(1 / \cos \beta) \int_{0}^{z} \bar{\alpha}_{n m}\left(z^{\prime}\right) d z^{\prime}}
$$

and a further integration over $\Omega$ results in

$$
\begin{array}{r}
I_{\nu}(z)=\int_{0}^{2 \pi} \int_{0}^{\pi / 2} I_{\nu, \Omega}(z) \cos \beta \sin \beta d \beta d \varphi \\
=I_{\nu, \Omega}(0) \int_{0}^{2 \pi} \int_{0}^{\pi / 2} e^{-(1 / \cos \beta) \int_{0}^{z} \bar{\alpha}_{n m}\left(z^{\prime}\right) d z^{\prime}} \\
\quad \times \cos \beta \sin \beta d \beta d \varphi .
\end{array}
$$

First integrating over $\varphi$ and introducing the optical depth $\int_{0}^{z} \bar{\alpha}_{n m}\left(z^{\prime}\right) d z^{\prime}=\tau$ as well as the substitution $u=\tau / \cos \beta$, we can write

$$
\begin{aligned}
I_{\nu}(\tau) & =2 \pi I_{\nu, \Omega}(0) \tau^{2} \int_{\tau}^{\infty} \frac{e^{-u}}{u^{3}} d u \\
& =I_{\nu}(0) \tau^{2}\left(E_{1}(\tau)+e^{-\tau}\left(\frac{1}{\tau^{2}}-\frac{1}{\tau}\right)\right)
\end{aligned}
$$

with $I_{\nu}(0)=\pi I_{\nu, \Omega}(0)$ (see (51)) and the exponential integral

$$
E_{1}(\tau)=\int_{\tau}^{\infty} \frac{e^{-u}}{u} d u=-0.5772-\ln \tau+\sum_{k=1}^{\infty} \frac{(-1)^{k+1} \tau^{k}}{k \cdot k !}
$$

Since the further considerations in this paper are concentrating on the radiation transfer in the atmosphere under the influence of thermal background radiation, it is more appropriate to describe the interaction of radiation with the molecules by a stepwise propagation through thin layers of depth $\Delta z$ as given by (55).

3.3. Rate Equations under Atmospheric Conditions. As a further generalization of the rate equations (35), in this subsection, we additionally consider the influence of the external radiation, which together with the thermal background radiation is acting on the molecules in the presence of collisions (see Figure 8).

And different to Section 2, the infrared active molecules are considered as a trace gas in an open system, the atmosphere, which has to come into balance with its environment. Then molecules radiating due to their temperature and thus loosing part of their energy have to get this energy back from the surrounding by IR radiation, by sensitive or latent heat or also by absorption of sunlight. This is a consequence of energy conservation.

The radiation loss is assumed to be proportional to the actual photon density $\rho_{G}$ and scaling with a flux rate $\phi$. This loss can be compensated by the absorbed power of the incident radiation, for example, the terrestrial radiation, which is further expressed in terms of the photon density $\rho_{E}$ (see (57)), and it can also be replaced by thermal energy. Therefore, the initial rate equations have to be supplemented by additional relations for these two processes.

It is evident that both, the incident radiation and the heat flux, will be limited by some genuine interactions. So, the radiation can only contribute to a further excitation, as long as it is not completely absorbed. At higher molecular densities, however, the penetration depth of the radiation in the gas is decreasing and therewith also the effective excitation over some longer volume element. Integrating (55) over the linewidth while using the definitions of (31) and (32) and then integrating over $z$ results in

$$
I_{\nu_{m n}}(z)=I_{\nu_{m n}}(0) e^{-2 S_{n m} N z / \Delta v}
$$

which due to Lambert-Beer's law describes the averaged spectral intensity over the linewidth as a function of the propagation in $z$-direction. From this, it is quite obvious to define the penetration depth as the length $L_{P}$, over which the initial intensity reduces to $1 / e$ and thus the exponent in (67) gets unity with

$$
L_{P}=\frac{\Delta \nu}{2 S_{n m} N_{S}} .
$$

From (68), also the molecular number density $N_{S}$ is found, at which the initial intensity just drops to $1 / e$ after an interaction length $L_{P}$. Since $N_{S}$ characterizes the density at which an excitation of the gas gradually comes to an end and in this sense saturation takes place, it is known as the saturation density, where $N_{S}$ and also $S_{n m}$ refer to the total number density $N$ of the gas.

Since at constant pressure, the number density in the gas is changing with the actual temperature due to GayLussac's law and the molecules are distributed over hundreds of states and substates, the molecular density, contributing to an interaction with the radiation on the transition $n \rightarrow m$, is given by [15]

$$
N_{n}=N_{0} \frac{T_{0}}{T_{G}} \frac{g_{n} e^{-E_{n} / k T_{G}}}{P\left(T_{G}\right)},
$$

where $N_{0}$ is the molecular number density at an initial temperature $T_{0}, E_{n}$ is the energy of the lower level $n$ above 
the ground state, and $P\left(T_{G}\right)$ is the total internal partition sum, defined as $[15,16]$

$$
P\left(T_{G}\right)=\sum_{i} g_{i} e^{-E_{i} / k T_{G}}
$$

In the atmosphere typical propagation, lengths are of the order of several $\mathrm{km}$. So, for a stronger $\mathrm{CO}_{2}$ transition in the $15 \mu \mathrm{m}$ band with a spontaneous rate $A=1 \mathrm{~s}^{-1}, N_{n} / N=$ $N_{n} / N_{0}=0.07$, a spectral line intensity $S_{n m}$ or integrated cross section of

$$
\begin{aligned}
S_{n m} & =\bar{\sigma}_{n m}^{0}=\frac{A_{m n} \lambda^{2}}{8 \pi n^{2}} \frac{N_{n}}{N}\left(1-\frac{g_{n}}{g_{m}} \frac{N_{m}}{N_{n}}\right) \\
& =6 \times 10^{-13} \mathrm{~m}^{2} \mathrm{~Hz}=2 \times 10^{-19} \mathrm{~cm}^{-1} /\left(\text { molecules } \cdot \mathrm{cm}^{-2}\right)
\end{aligned}
$$

and a spectral width at ground pressure of $\Delta v=5 \mathrm{GHz}$, the saturation density over a typical length of $L_{P}=1 \mathrm{~km}$ assumes a value of $N_{S} \approx 4 \times 10^{18}$ molecules $/ \mathrm{m}^{3}$. Since the number density of the air at $1013 \mathrm{hPa}$ and $288 \mathrm{~K}$ is $N_{\text {air }}=$ $2.55 \times 10^{25} \mathrm{~m}^{-3}$, saturation on the considered transition already occurs at a concentration of less than one ppm. It should also be noticed that at higher altitudes and thus lower densities, also the linewidth $\Delta \nu$ due to pressure broadening reduces. This means that to first order, also the saturation density decreases with $\Delta \nu$, while the gas concentration in the atmosphere, at which saturation appears, almost remains constant. The same applies for the penetration depth.

For the further considerations, it is adequate to introduce an average spectral intensity $\bar{I}_{v_{m n}}$ as

$$
\begin{aligned}
\bar{I}_{\nu_{m n}} & =\frac{1}{L_{P}} \int_{0}^{L_{P}} I_{v_{m n}}(z) d z \\
& =\frac{\Delta v}{2 S_{n m} N L_{P}} I_{v_{m n}}(0) \cdot\left(1-e^{-2 S_{n m} N L_{P} / \Delta v}\right)
\end{aligned}
$$

or equivalently an average photon density

$$
\bar{\rho}_{E}=\frac{n}{c} \frac{\Delta \nu}{h \nu_{m n}} \bar{I}_{v_{m n}}=\frac{\Delta \nu}{2 S_{n m} N L_{P}} \rho_{E}(0) \cdot\left(1-e^{-2 S_{n m} N L_{P} / \Delta v}\right)
$$

which characterizes the incident radiation with respect to its average excitation over $L_{P}$ in the rate equations. In general, the incident flux $\rho_{E}(0)$ onto an atmospheric layer consists of two terms, the up- and the downwelling radiation.

Any heat flux, supplied to the gas volume, contributes to a volume expansion, and via collisions and excitation of molecules, this can also be transferred to radiation energy. Simultaneously absorbed radiation can be released as heat in the gas. Therefore, the rate equations are additionally supplemented by a balance for the heat energy density of the air $Q\left[\mathrm{~J} / \mathrm{m}^{3}\right]$, which under isobaric conditions and making use of the ideal gas equation in the form $p_{\text {air }}=N_{\text {air }} \cdot k \cdot T_{G}$ can be written as

$$
\begin{aligned}
\Delta Q & =c_{p} \rho_{\text {air }} \Delta T_{G}=\left(\frac{f}{2}+1\right) \frac{R_{G}}{M_{\text {air }}} \frac{M_{\text {air }}}{N_{A}} \frac{p_{\text {air }}}{k T_{G}} \Delta T_{G} \\
& =\frac{7}{2} \frac{p_{\text {air }}}{T_{G}} \Delta T_{G},
\end{aligned}
$$

or after integration

$$
Q\left(T_{G}\right)=\frac{7}{2} p_{\text {air }} \int_{T_{0}}^{T_{G}} \frac{1}{T_{G}^{\prime}} d T_{G}^{\prime}=\frac{7}{2} p_{\text {air }} \ln \left(\frac{T_{G}}{T_{0}}\right) .
$$

Here $c_{p}$ represents the specific heat capacity of the air at constant pressure with $c_{p}=(f / 2+1) \cdot R_{G} / M_{\text {air }}$, where $f$ are the degrees of freedom of a molecule (for $\mathrm{N}_{2}$ and $\mathrm{O}_{2}: f=5$ ) and $R_{G}=k \cdot N_{A}$ is the universal gas constant (at room temperature: $\left.c_{p}=1.01 \mathrm{~kJ} / \mathrm{kg} / \mathrm{K}\right)$. $\rho_{\text {air }}$ is the specific weight of the air (at room temperature and ground pressure: $\rho_{\text {air }}=$ $1.29 \mathrm{~kg} / \mathrm{m}^{3}$ ), which can be expressed as $\rho_{\text {air }}=m_{\text {air }} \cdot N_{\text {air }}=$ $M_{\text {air }} / N_{A} \cdot N_{\text {air }}=M_{\text {air }} / N_{A} \cdot p_{\text {air }} /\left(k T_{G}\right)$ with $m_{\text {air }}$ as the mass of an air molecule, $M_{\text {air }}$ the mol weight, $N_{A}$ Avogadro's number, $N_{\text {air }}$ the number density, and $p_{\text {air }}$ the pressure of the air.

The thermal energy can be supplied to a volume element by different means. So, thermal convection and conductivity in the gas contribute to a heat flux $\mathbf{j}_{H}\left[\mathrm{~J} /\left(\mathrm{s} \cdot \mathrm{m}^{2}\right)\right]$, causing a temporal change of $Q$ proportional to $\operatorname{div} j_{H}$, or for one direction proportional to $\partial j_{H z} / \partial z$. $\mathbf{j}_{H}$ is a vector and points from hot to cold. Since this flux is strongly dominated by convection, it can be well approximated by $j_{\mathrm{Hz}}=h_{\mathrm{C}} \cdot\left(T_{E}-\right.$ $T_{G}$ ) with $h_{C}$ as the heat transfer coefficient, typically of the order of $h_{C} \approx 10-15 \mathrm{~W} / \mathrm{m}^{2} / \mathrm{K}$. At some more or less uniform distribution of the incident heat flux over the troposphere (altitude $H_{T} \approx 12 \mathrm{~km}$ ), the temporal change of $Q$ due to convection may be expressed as $\partial j_{H z} / \partial z=h_{C} / H_{T} \cdot\left(T_{E}-T_{G}\right)$ with $h_{C} / H_{T} \approx 1 \mathrm{~mW} / \mathrm{m}^{3} / \mathrm{K}$.

Another contribution to the thermal energy originates from the absorbed sunlight, which in the presence of collisions is released as kinetic or rotational energy of the molecules. Similarly, latent heat can be set free in the air. In the rate equations, both contributions are represented by a source term $q_{\mathrm{SL}}\left[\mathrm{J} /\left(\mathrm{s} \cdot \mathrm{m}^{3}\right)\right]$.

Finally, the heat balance is determined by exciting and deexciting collisions, changing the population of the states $n$ and $m$, and reducing or increasing the energy by $h \cdot v_{m n}$.

Altogether, this results in a set of coupled differential equations, which describe the simultaneous interaction of molecules with their self-generated thermal background radiation as well as with radiation from the earth's surface 
and/or a neighbouring layer, this all in the presence of collisions and under the influence of heat transfer processes:

$$
\begin{aligned}
\frac{d N_{m}}{d t}= & +\left(\frac{h v_{m n}}{\Delta v} B_{n m}\left(\rho_{G}+\bar{\rho}_{E}\right)+C_{n m}\right) N_{n} \\
& -\left(\frac{h \nu_{m n}}{\Delta v} B_{m n}\left(\rho_{G}+\bar{\rho}_{E}\right)+C_{m n}+A_{m n}\right) N_{m}, \\
\frac{d N_{n}}{d t}=- & \left(\frac{h v_{m n}}{\Delta v} B_{n m}\left(\rho_{G}+\bar{\rho}_{E}\right)+C_{n m}\right) N_{n} \\
& +\left(\frac{h v_{m n}}{\Delta v} B_{m n}\left(\rho_{G}+\bar{\rho}_{E}\right)+C_{m n}+A_{m n}\right) N_{m}, \\
\frac{d \rho_{G}}{d t}=- & \frac{h v_{m n}}{\Delta v}\left(B_{n m} N_{n}-B_{m n} N_{m}\right) \rho_{G}+A_{m n} N_{m}-\phi \rho_{G}, \\
\frac{d \bar{\rho}_{E}}{d t}=+ & \frac{c / n}{2 L_{P}}\left(1-e^{-\left(2 h v_{m n} /(\Delta v c / n)\right)\left(B_{n m} N_{n}-B_{m n} N_{m}\right) L_{P}}\right) \rho_{E}(0) \\
& -\frac{h v_{m n}}{\Delta v}\left(B_{n m} N_{n}-B_{m n} N_{m}\right) \bar{\rho}_{E}, \\
\frac{d Q}{d t}=+ & \frac{h C}{H_{T}}\left(T_{E}-T_{G}\right)+q_{\mathrm{SL}}-h v_{m n}\left(C_{n m} N_{n}-C_{m n} N_{m}\right) .
\end{aligned}
$$

To symbolize the mutual coupling of these equations, here we use a notation where the radiation induced transition rates are represented by the Einstein coefficients and the respective photon densities. A change to the other representation is easily performed applying the identities:

$$
\begin{gathered}
W_{m n}^{G}=\frac{h v_{m n}}{\Delta v} B_{m n} \rho_{G}=B_{m n} u_{v_{m n}} \\
W_{m n}^{E}=\frac{h v_{m n}}{\Delta v} B_{m n} \rho_{E}=B_{m n} \frac{n}{c} I_{v_{m n}}, \\
W_{n m}^{G, E}=\frac{g_{m}}{g_{n}} W_{m n}^{G, E} .
\end{gathered}
$$

It should be noticed that the rate equation for $\bar{\rho}_{E}$ could also be replaced by the incident radiation, as given by (73). However, since $\bar{\rho}_{E}$ approaches its equilibrium value within a time constant $\tau=\Delta \nu /\left(h \nu_{m n}\left(B_{n m} N_{n}-B_{m n} N_{m}\right)\right)$ short compared with other processes, for a uniform representation the differential form was preferred.

For the special case of stationary equilibrium with $d N_{n} / d t=d N_{m} / d t=0$ from the first rate equation, we get for the population ratio:

$$
\begin{aligned}
\frac{N_{m}}{N_{n}} & =\frac{h v_{m n} B_{n m}\left(\rho_{G}+\bar{\rho}_{E}\right) / \Delta \nu+C_{n m}}{h \nu_{m n} B_{m n}\left(\rho_{G}+\bar{\rho}_{E}\right) / \Delta \nu+C_{m n}+A_{m n}} \\
& =\frac{W_{n m}^{G}+W_{n m}^{E}+C_{n m}}{W_{m n}^{G}+W_{m n}^{E}+A_{m n}+C_{m n}}
\end{aligned}
$$

which at $T_{G} \sim T_{E}=288 \mathrm{~K}$ and $\lambda=15 \mu \mathrm{m}$ due to $W_{m n}^{G}=$ $4 W_{m n}^{E}=0.037 A_{m n}$ (see (37) and (60)) in the limit of pure spontaneous decay processes reaches its maximum value of $4.4 \%$, while in the presence of collisions with collision rates in the troposphere of several $10^{9} \mathrm{~s}^{-1}$, it rapidly converges to $3.5 \%$, given by the Boltzmann relation at temperature $T_{G}$ and corresponding to a local thermodynamic equilibrium.

For the general case, the rate equations have to be solved numerically, for example, by applying the finite element method. While (76) in the presented form is only valid for a two-level system, a simulation under realistic conditions comparable to the atmosphere requires some extension, particularly concerning the energy transfer from the earth's surface to the atmosphere. Since the main trace gases $\mathrm{CO}_{2}$, water vapour, methane, and ozone are absorbing the incident infrared radiation simultaneously on thousands of transitions, as an acceptable approximation for this kind of calculation, we consider these transitions to be similar and independent from each other, each of them contributing to the same amount to the energy balance. In the rate equations, this can easily be included by multiplying the last term in the equation for the energy density $Q$ with an effective number $M_{\text {tr }}$ of transitions.

In this approximation, the molecules of an infrared active gas and even a mixture of gases are represented by a "standard transition" which reflects the dynamics and time evolution of the molecular populations under the influence of the incident radiation, the background radiation, and the thermal heat transfer. $M_{\mathrm{tr}}$ is derived as the ratio of the total absorbed infrared intensity over the considered propagation length to the contribution of a single standard transition.

An example for a numerical simulation in the troposphere, more precisely for a layer from ground level up to $100 \mathrm{~m}$, is represented in Figure 9. The graphs show the evolution of the photon densities $\rho_{G}$ and $\bar{\rho}_{E}$, the population densities $N_{n}$ and $N_{m}$ of the states $n$ and $m$, the accumulated heat density $Q$ in the air, and the temperature $T_{G}$ of the gas (identical with the atmospheric temperature) as a function of time and as an average over an altitude of $100 \mathrm{~m}$.

As initial conditions we assumed a gas and air temperature of $T_{G}=40 \mathrm{~K}$, a temperature of the earth's surface of $T_{E}=288 \mathrm{~K}$, an initial heat flux due to convection of $j_{H z}=3 \mathrm{~kW} / \mathrm{m}^{2}$, a latent heat source of $q_{\mathrm{SL}}=3 \mathrm{~mW} / \mathrm{m}^{3}$, and an air pressure of $p_{\text {air }}=1013 \mathrm{hPa}$. The interaction of the terrestrial radiation with the infrared active gases is demonstrated for $\mathrm{CO}_{2}$ with a concentration in air of $380 \mathrm{ppm}$ corresponding to a molecular number density of $N_{0}=$ $9.7 \times 10^{21} \mathrm{~m}^{-3}$ at $288 \mathrm{~K}$. The simulation was performed for a "standard transition" as discussed before with a transition wavelength $\lambda=15 \mu \mathrm{m}$, a spontaneous transition rate $A_{m n}=$ $1 \mathrm{~s}^{-1}$, statistical weights of $g_{m}=35$ and $g_{n}=33$, a spectral width of $\Delta v=5 \mathrm{GHz}$, a collisional transition rate $C_{m n}=10^{8} \mathrm{~s}^{-1}$ (we do not distinguish between rotational and vibrational rates [10]), a penetration depth $L_{P}=100 \mathrm{~m}$, and assuming a photon loss rate of $\phi=7.5 \times 10^{5} \mathrm{~s}^{-1}$. For this calculation, a density and temperature dependence of $\Delta v$ and $C_{m n}$ was neglected. Since under these conditions a single transition contributes $0.069 \mathrm{~W} / \mathrm{m}^{2}$ to the total IR-absorption of $212 \mathrm{~W} / \mathrm{m}^{2}$ over $100 \mathrm{~m}, M_{\mathrm{tr}}=3095$ transitions represent the radiative interaction with all active gases in the atmosphere. 


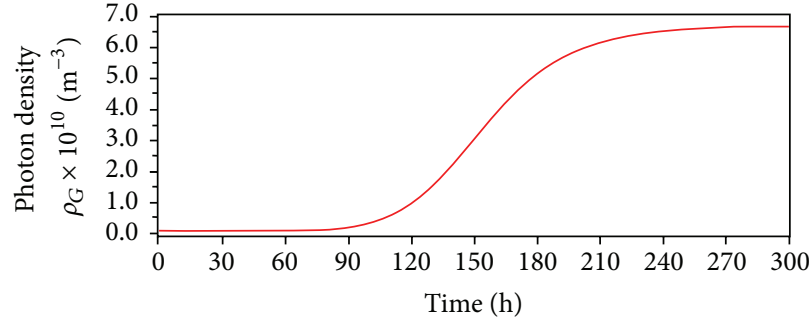

(a)

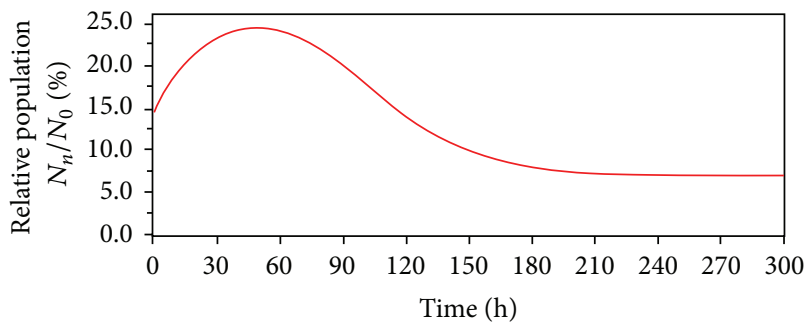

(c)

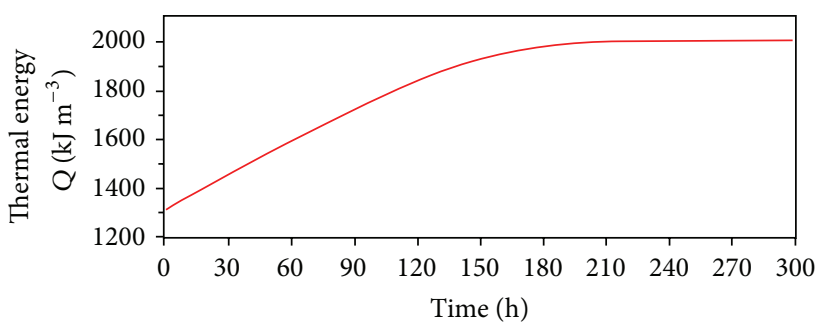

(e)

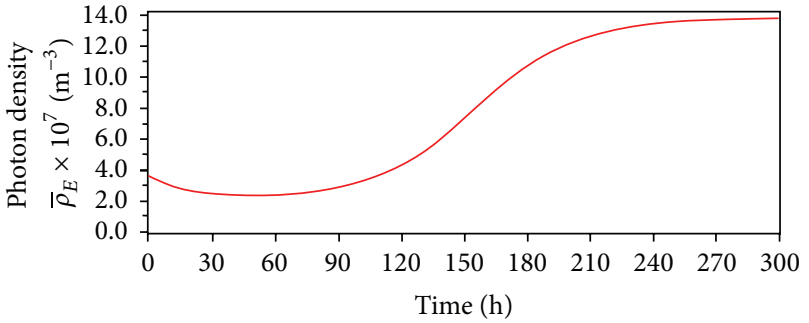

(b)

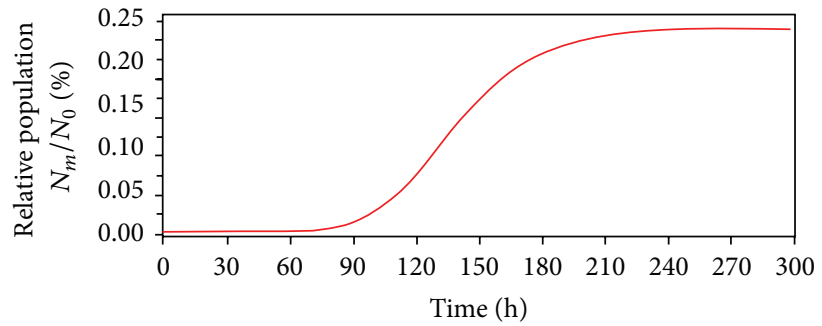

(d)

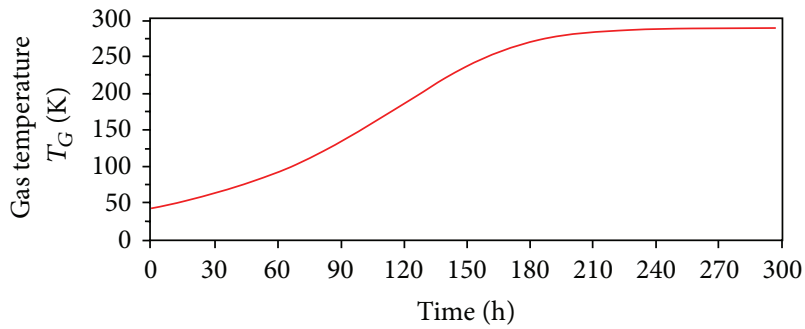

(f)

FIGURE 9: Solution of the rate equations for the troposphere (close to the earth's surface) representing the evolution of (a) the photon density $\rho_{G}$ (b) the photon density $\bar{\rho}_{E}$ (c) the lower and (d) the upper population density, (e) the thermal energy density, and (f) the gas- (air)-temperature as a function of time.

The evolution for another gas and transition can easily displayed by replacing the respective parameters for the other transition and recalibrating the effective transition number.

To ensure reproducible results and to avoid any instabilities, the time interval for the stepwise integration of the coupled differential equation system has to be chosen small enough to avoid changes comparable to the size of a computed quantity itself, or the calculated changes have to be restrict by some upper boundaries, taking into account some smaller repercussions on the absolute time scale. The latter procedure was applied which accelerates the calculations by several orders of magnitude.

A simulation is done in such a way, that starting from the initial conditions for the populations, the photon densities, and the temperature, in a first step, modified population and photon densities as well as a change of the heat density over the time interval $\Delta t$ are determined. The heat change causes a temperature change $\Delta T_{G}$ (see (74)) and gives a new temperature, which is used to calculate a new collision transfer rate $C_{n m}$ by (36), a corrected population $N_{n}$ by (69) and a new population $N_{m}$ for the upper state by (4). The total internal partition sum for $\mathrm{CO}_{2}$ is deduced from data of the
HITRAN-database [13] and approximated by a polynomial of the form:

$$
P\left(T_{G}\right)=0.894 \cdot T_{G}+3 \times 10^{-9} T_{G}^{4} .
$$

The new populations are used as new initial conditions for the next time step, over which again the interaction of the molecules with the radiation and any heat transfer is computed. In this way, the time evolution of all coupled quantities shown in Figure 9 is derived. For this simulation, it was assumed that all atmospheric components still exist in gas form down to $40 \mathrm{~K}$.

The relatively slow evolution of the curves is due to the fact, that the absorbed IR radiation as well as the thermal flux is mostly used to heat up the air volume which at constant air pressure is further expanding and the density decreasing.

Population changes of the $\mathrm{CO}_{2}$ states, induced by the external or the thermal background radiation, are coupled to the heat reservoir via the collisional transition rates $C_{m n}$ and $C_{n m}$ (see last rate equation), which are linked to each other by (36). As long as the calculated populations differ from a Boltzmann distribution at the gas-temperature $T_{G}$, 
the two terms in the parenthesis of this rate equation do not compensate, and their difference contributes to an additional heating, amplified by the effective number of transitions. Reasons for smaller deviations from a local thermal equilibrium can be the induced population changes due to the external radiation, as well as temperature and density variations with altitude and daytime, or some local effects in the atmosphere. Also vice versa, when the gas is supplied with thermal energy, the heat can be transferred to electromagnetic energy and reradiated by the molecules (radiative cooling).

The photon density $\rho_{G}$ (Figure $9(\mathrm{a})$ ) shows the spontaneous emission of the molecules and determines the thermal background radiation of the gas. It is mostly governed by the population of the upper state and in equilibrium is selfadjusting on a level where the spontaneous generation rate $A_{m n} \cdot N_{m}$ is just balanced by the induced transition rates (optical and collision induced) and the loss rate $\phi \rho_{G}$, the latter dependent on the density and temperature variations in the atmosphere.

Figure 9 (b) represents the effective photon density $\bar{\rho}_{E}$ available to excite the gas over the layer depth $L_{P}$ by terrestrial radiation and back radiation from the overlaying atmosphere. The downwelling part was assumed to originate from a layer of slightly lower temperature than the actual gas temperature $T_{G}$ with a lapse rate also varying with $T_{G}$. The curve first declines, since with slightly increasing temperature, the lower state, which is not the ground state of $\mathrm{CO}_{2}$, is stronger populated (see also Figure 9(c)), and therefore the absorption on this transition increases, while according to saturation effects, the effective excitation over $L_{P}$ decreases. With further growing temperature $\bar{\rho}_{E}$ again increases, caused by the decreasing gas density and also reduced lower population, accompanied by a smaller absorption.

Figures 9(c) and 9(d) show the partial increase as well as the depletion of the lower state and the simultaneous refilling of the upper state due to the radiation interaction and thermal heating. The populations are displayed in relative units, normalized to the $\mathrm{CO}_{2}$ number density $N_{0}$ at $288 \mathrm{~K}$.

The heat density of the air and the gas (air) temperature, are plotted in Figures 9(e) and 9(f). Up to about $80 \mathrm{~K}$ (first $70 \mathrm{~h}$ ), heating is determined by convection, while at higher temperatures radiative heating more and more dominates. An initial heat flux of $3 \mathrm{~kW} / \mathrm{m}^{2}$ seems relatively large but quickly reduces with increasing temperature and at a difference of $3 \mathrm{~K}$ between the surface and lower atmosphere, similar to latent heat, does not contribute to more than $40 \mathrm{~W} / \mathrm{m}^{2}$, representing only $10 \%$ of the terrestrial radiation.

In principle, it is expected that a gas heated by an external source cannot get warmer than the source itself. This is valid for a heat flux caused by convection or conduction in agreement with the second law of thermodynamics, but it leads to some contradiction with respect to a radiation source.

In a closed system as discussed in Section 2, thermal radiation interacting with the molecules is in unison with the population of the molecules, and both the radiation and the gas can be characterized by a unique temperature $T_{G}$. In an open system with additional radiation from an external source of temperature $T_{E}$, the prevailing radiation, interacting with the molecules, consists of two contributions, which in general are characterized by different temperatures and different loss processes. So, $T_{E}$ determines the spectral intensity and distribution of the incident radiation and in this sense defines the power flux which can be transferred to the molecules, when this radiation is absorbed. But it does not describe any direct heat transfer, which always requires a medium for the transport and by no means defines the temperature of the absorbing molecules. At thermal equilibrium and in the presence of collisions, the molecules are better described by a temperature, deduced from the population ratio $N_{m} / N_{n}$ given by the Boltzmann relation (4). This temperature also determines the spontaneous emission and together with the losses defines the thermal background radiation.

Since an absorption on a transition takes place as long as the population difference $N_{n}-N_{m}$ is positive (equal populations signify infinite temperature), molecules can be well excited by a thermal radiation source, which has a temperature lower than that of the absorbing gas. Independent of this statement, it is clear that at equilibrium the gas can only reradiate that amount of energy that was previously absorbed from the incident radiation and/or sucked up as thermal energy.

In any way, for the atmospheric calculations, it seems reasonable, to restrict the maximum gas temperature to values comparable to the earth's surface temperature. An adequate condition for this is a radiation loss rate

$$
\phi \geq \frac{c / n}{4 L_{P}}\left(1-e^{-\left(2 h v_{m n} /(\Delta v c / n)\right)\left(B_{n m} N_{n}-B_{m n} N_{m}\right) L_{P}}\right),
$$

as derived from the rate equations at stationary equilibrium and with up- and downwelling radiation of the same magnitude.

A calculation for conditions as found in the upper stratosphere at about $60 \mathrm{~km}$ altitude is shown in Figure 10. The air pressure is $0.73 \mathrm{hPa}$, the regular temperature at this altitude is $242.7 \mathrm{~K}$, and the number density of air is $N_{\text {air }}=2.2 \times 10^{22} \mathrm{~m}^{-3}$. The density of $\mathrm{CO}_{2}$ reduces to $N=8.3 \times 10^{18} \mathrm{~m}^{-3}$ at a concentration of $380 \mathrm{ppm}$. At this lower pressure, the collision induced linewidth is three orders of magnitude smaller and declines to about $\Delta v=5 \mathrm{MHz}$. Under these conditions, the remaining width is governed by Doppler broadening with $34 \mathrm{MHz}$. At this altitude, any terrestrial radiation for an excitation on the transition is no longer available. The only radiation interacting with the molecules originates from neighbouring molecules and the own background radiation.

As initial conditions we used a local gas and air temperature $T_{G}=250 \mathrm{~K}$, slightly higher than the environment with $T_{E}=242.7 \mathrm{~K}$, the latter defining the radiation (up and down) from neighbouring molecules. The heat flux $j_{\mathrm{Hz}}$ from or to adjacent layers was assumed to be negligible, while a heat well due to sun light absorption by ozone with $\sim 15 \mathrm{~W} / \mathrm{m}^{2}$ over $\sim 50 \mathrm{~km}$ in this case is the only heat source with $q_{\mathrm{SL}}=$ $0.3 \mathrm{~mW} / \mathrm{m}^{3}$. The calculation was performed for a collisional transition rate $C_{m n}=10^{5} \mathrm{~s}^{-1}$, a photon loss rate of $\phi=$ $7.5 \times 10^{4} \mathrm{~s}^{-1}$, a layer depth of $L_{P}=1 \mathrm{~km}$, and an effective number of transitions $M_{\mathrm{tr}}=1430$ (absorption on standard 


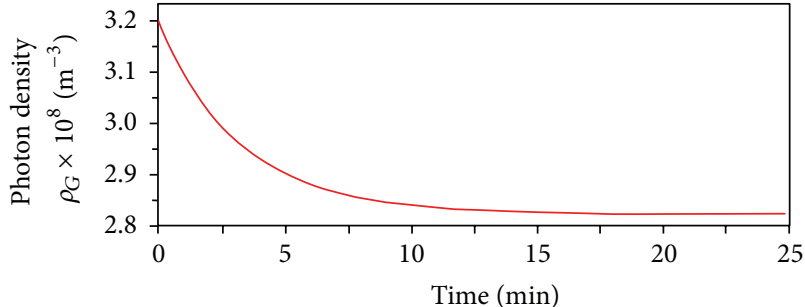

(a)

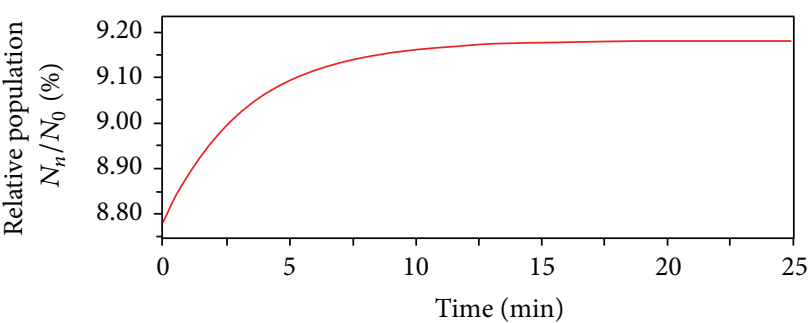

(c)

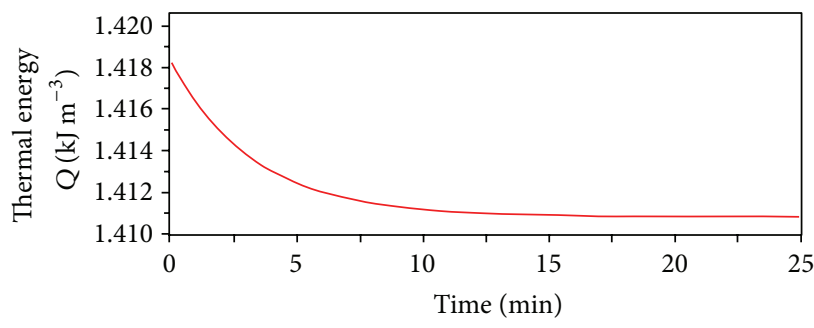

(e)

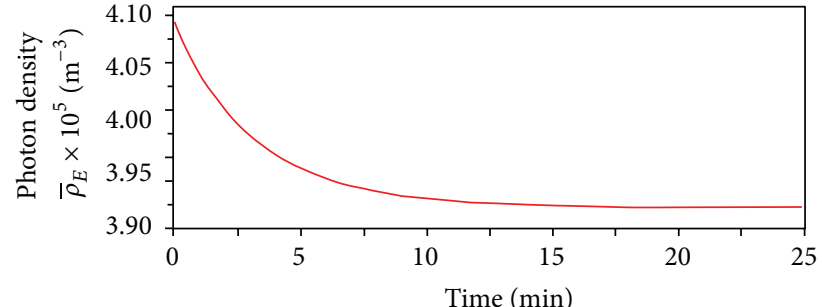

(b)

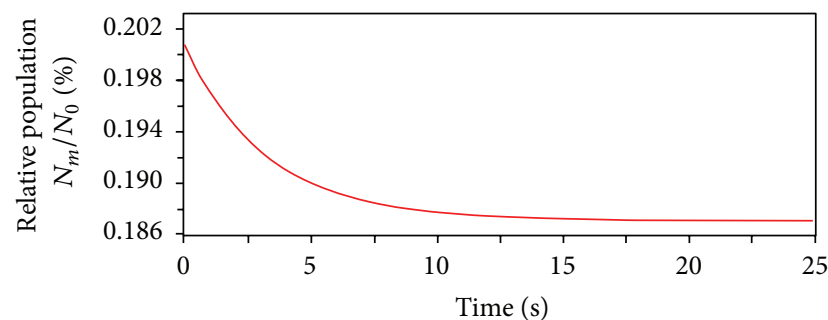

(d)

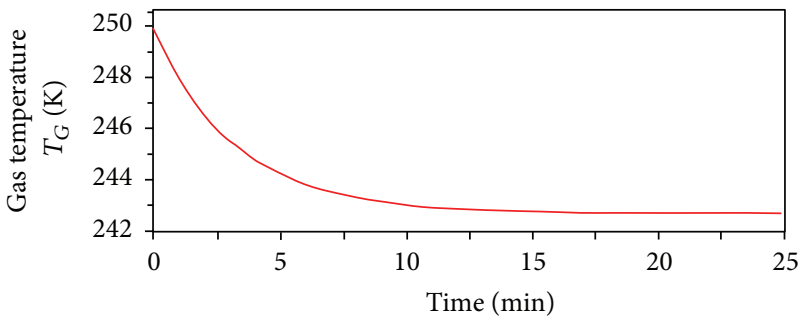

(f)

FIGURE 10: Solution of the rate equations for the stratosphere $\left(60 \mathrm{~km}\right.$ altitude) representing the evolution of (a) the photon density $\rho_{G}$, (b) the photon density $\bar{\rho}_{E}$, (c) the lower and (d) the upper population density, (e) the thermal energy density, and (f) the gas (atmospheric) temperature as a function of time.

transition: $2.8 \times 10^{-4} \mathrm{~W} / \mathrm{m}^{2}$; total IR-absorption over $1 \mathrm{~km}$ : $\left.0.4 \mathrm{~W} / \mathrm{m}^{2}\right)$.

Similar to Figure 9, the graphs show the evolution of the photon densities $\rho_{G}$ and $\bar{\rho}_{E}$, the population densities $N_{n}$ and $N_{m}$ of the states $n$ and $m$, the heat density $Q$ in air, and the temperature $T_{G}$ of the gas as a function of time. But different to the conditions in the troposphere, where heating of the atmosphere due to terrestrial heat and radiation transfer was the dominating effect, the simulations in Figure 10 demonstrate the effect of radiative cooling in the stratosphere, where incident radiation from adjacent layers and locally released heat is transferred to radiation and radiated to space. An increased reradiation will be observed, as represented by the upper graph till the higher temperature diminishes and the radiated energy is in equilibrium with the supplied energy flux.

Due to the lower density and heat capacity of the air at this reduced pressure, the system comes much faster to a stationary equilibrium.

3.4. Thermodynamic and Radiation Equilibrium. Thermodynamic equilibrium in a gas sample means that the population of the molecular states is given by the Boltzmann distribution (4) and the gas is characterized by an average temperature $T_{G}$. On the other hand, spectral radiation equilibrium requires that the number of absorptions in a considered spectral interval is equal to the number of emissions. While without an external radiation generally, any thermodynamic equilibrium in a sample will be identical with the spectral radiation equilibrium, in the presence of an additional field, these cases have to be distinguished, since the incident radiation also induces transitions and modifies the populations of the molecular states, deviating from the Boltzmann distribution.

Therefore, it is appropriate to investigate the population ratio more closely and to discover how it looks like under some special conditions. This is an adequate means to determine what kind of equilibrium is found in the gas sample.

For this, we consider the total balance of absorption and emission processes as given by the rate equations (76). Under stationary equilibrium conditions (see (78)), it is

$$
\left(W_{n m}^{G}+W_{n m}^{E}+C_{n m}\right) N_{n}=\left(W_{m n}^{G}+W_{m n}^{E}+A_{m n}+C_{m n}\right) N_{m} .
$$


With (17), (34) and (59) we can write

$$
\begin{aligned}
& \left(B_{n m}\left(u_{v}+\frac{n}{c} \cdot I_{v}\right)+C_{n m}\right) N_{n} \\
& \quad=\left(B_{m n}\left(u_{v}+\frac{n}{c} \cdot I_{v}\right)+A_{m n}+C_{m n}\right) N_{m} .
\end{aligned}
$$

At local thermodynamic equilibrium, $C_{m n}$ and $C_{n m}$ are linked to each other by (36). But even more generally, we can conclude that (36) is valid, as long as the molecules can be described by a Maxwellian velocity distribution and can be characterized by an average temperature (see also [12]).

Thus, applying (36) and introducing some abbreviations:

$$
\begin{aligned}
& u_{v}+\frac{n}{c} \cdot I_{v}=U_{v}, \quad \frac{C_{n m}}{B_{n m}}=\varepsilon, \\
& \frac{A_{m n}}{B_{n m}}=\frac{g_{n}}{g_{m}} \frac{8 \pi n^{2}}{\lambda_{m n}^{2}} \frac{h v_{m n}}{c / n}=\frac{g_{n}}{g_{m}} \kappa
\end{aligned}
$$

after elementary rearrangement, (82) becomes:

$$
\frac{N_{m}}{N_{n}}=\frac{g_{m}}{g_{n}} \frac{U_{v}+\varepsilon}{U_{v}+\kappa+\varepsilon \cdot e^{h v_{m n} /\left(k T_{G}\right)}} .
$$

It is easy to be seen that for large $\varepsilon$ and thus high collision rates, (84) approaches to a Boltzmann distribution. Also for $\varepsilon=0$ (no collisions) and $I_{v}=0$ (no external radiation), this gives the Boltzmann relation, while for $\varepsilon=0$ and $I_{v} \gg(c / n)$. $u_{v}$, (84) changes to

$$
\begin{aligned}
\frac{N_{m}}{N_{n}} & \approx \frac{g_{m}}{g_{n}} \frac{I_{\nu}}{I_{\nu}+(c / n) \kappa}=\frac{g_{m}}{g_{n}} \frac{1}{1+4\left(e^{h v_{m n} /\left(k T_{E}\right)}-1\right) e^{2 S_{n m} N z / \Delta v}} \\
& \approx \frac{1}{4} \frac{g_{m}}{g_{n}} e^{-h v_{m n} / k T_{E}} e^{-2 S_{n m} N z / \Delta v} .
\end{aligned}
$$

This equation shows that under conditions as found in the upper atmosphere (small collision rates), the populations are approximated by a modified Boltzmann distribution (right hand side: for $h v_{m n}>k T_{E}$ ), which with increasing spectral intensity is more and more determined by the temperature $T_{E}$ of the external radiation source. Under such conditions, the normal thermodynamic equilibrium according to (4) is violated and has to be replaced by the spectral radiation equilibrium.

\section{Radiation Balance and Radiation Transfer in the Atmosphere}

A more extensive analysis of the energy and radiation balance of an absorbing sample not only accounts for the net absorbed power from the incident radiation, as considered in (53)(66), but it also includes any radiation originating from the sample itself as well as any re-radiation due to the external excitation. This was already investigated in some detail in Section 3.3, however, with the view to a local balance and its evolution over time. In this section, we are particularly concentrating on the propagation of thermal radiation in an infrared active gas and how this radiation is modified due to the absorption and re-emission of the gas.

Two perspectives are possible, an energy balance for the sample or a radiation balance for the in- and outgoing radiation. Here we discuss the latter case. Scattering processes are not included.

We consider the incident radiation from an external source with the spectral radiance $I_{\nu, \Omega}\left(T_{E}\right)$ as defined in (50). Propagation losses in a thin gas layer obey Lambert-Beer's law. Simultaneously, this radiation is superimposed by the thermal radiation emitted by the gas sample itself, and as already discussed in Section 2.5, this thermal contribution has its origin in the spontaneous emission of the molecules. For small propagation distances $d r$ in the gas, both contributions can be summed up yielding

$$
\frac{d I_{\nu, \Omega}(r)}{d r}=-\bar{\alpha}_{n m}(\nu) I_{\nu, \Omega}\left(r, T_{E}\right)+\frac{h v_{m n}}{4 \pi} A_{m n} N_{m} g(\nu)
$$

The last term is a consequence of (19) or (45) when transforming the spectral energy density $u_{v}$ of the gas to a spectral intensity $(c / n) \cdot u_{v}$ and considering only that portion $(c / 4 \pi n)$. $u_{v}$ emitted into the solid angle interval $d \Omega$. Equation (86) represents a quite general form of the radiation balance in a thin layer, and it is appropriate to derive the basic relations for the radiation transfer in the atmosphere under different conditions. The differential $d r$ indicates that the absolute propagation length in the gas is meant.

4.1. Schwarzschild Equation in Dense Gases. In the troposphere, the population of molecular states is almost exclusively determined by collisions between the molecules even in the presence of stronger radiation. Then, due to the previous discussion, a well-established local thermodynamic equilibrium can be expected, and from (47) or (48), we find

$$
\frac{h v_{m n}}{4 \pi} A_{m n} N_{m} g(\nu)=\frac{c}{4 \pi n} \bar{\alpha}_{n m} u_{\nu}=\bar{\alpha}_{n m} B_{v, \Omega}\left(T_{G}\right)
$$

with $B_{v, \Omega}\left(T_{G}\right)=(c / 4 \pi n) \cdot u_{v}$ as the spectral radiance of the gas, also known as Kirchhoff-Planck-function and given by (50), but here at temperature $T_{G}$. To distinguish between the external radiation and the radiating gas, we use the letter $B$ (from background radiation), which may not be mixed with the Einstein coefficients $B_{n m}$ or $B_{m n}$. Then we get as the final result:

$$
\frac{d I_{\nu, \Omega}(r)}{d r}=-\bar{\alpha}_{n m}(\nu) I_{\nu, \Omega}\left(r, T_{E}\right)+\bar{\alpha}_{n m}(\nu) B_{\nu, \Omega}\left(T_{G}(r)\right) .
$$

This equation is known as the Schwarzschild equation [57], which describes the propagation of radiation in an absorbing gas and which additionally takes into account the thermal background radiation of the gas. While deriving this equation, no special restrictions or conditions concerning the density of the gas or collisions between the molecules have to be made, only that (36), is valid and the thermodynamic 


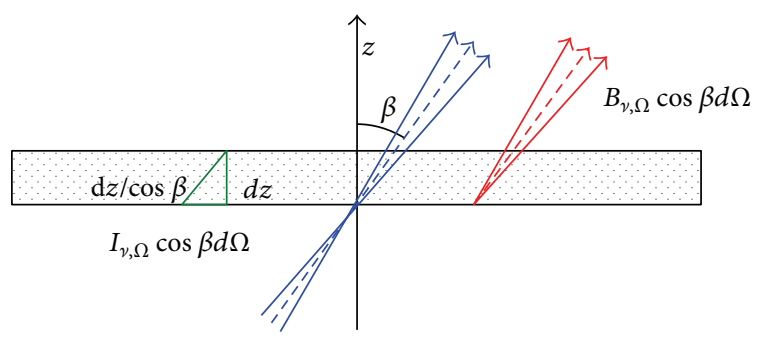

FIGURE 11: Radiation passing a gas layer.

equilibrium is given. In general, this is the case in the troposphere up to the stratosphere.

Another Derivation. Generally, the Schwarzschild equation is derived from pure thermodynamic considerations. The gas in a small volume is called to be in local thermodynamic equilibrium at temperature $T_{G}$, when the emitted radiation from this volume is the same as that of a blackbody radiator at this temperature. Then Kirchhoff's law is strictly valid, which means that the emission is identical to the absorption of a sample.

The total emission in the frequency interval $(\nu, \nu+d \nu)$ during the time $d t$ may be $4 \pi \varepsilon(\nu)$. The emissivity $\varepsilon(\nu)$ may not be mixed with $\varepsilon=C_{n m} / B_{n m}$ in (83). Then with the spectral radiance $B_{v, \Omega}\left(T_{G}\right)$ as given by (50) and with the absorption coefficient $\bar{\alpha}_{n m}(\nu)$, it follows from Kirchhoff's law (see, e.g., [8-11])

$$
\mathcal{\varepsilon}(\nu)=\bar{\alpha}_{n m}(\nu) \cdot B_{\nu, \Omega}\left(T_{G}\right)
$$

For an incident beam with the spectral radiance $I_{\nu, \Omega}$ and propagating in $r$-direction we then get:

$$
\begin{aligned}
\frac{d I_{\nu, \Omega}}{d r} & =-\bar{\alpha}_{n m}(\nu) I_{\nu, \Omega}\left(r, T_{E}\right)+\varepsilon(\nu) \\
& =-\bar{\alpha}_{n m}(\nu) I_{\nu, \Omega}\left(r, T_{E}\right)+\bar{\alpha}_{n m}(\nu) B_{\nu, \Omega}\left(T_{G}\right),
\end{aligned}
$$

which is identical with (88).

4.2. Radiation Transfer Equation for the Spectral Intensity. Normally the radiation and by this the energy, emitted into the full hemisphere, is of interest, so that (88) has to be integrated over the solid angle $\Omega=2 \pi$. As already discussed in Section 3.2, for this integration, we have to have in mind that a beam, propagating under an angle $\beta$ to the layer normal (see Figure 11), only contributes an amount $I_{\nu, \Omega} \cos \beta d \Omega$ to the spectral intensity (spectral flux density) due to Lambert's law. The same is assumed to be true for the thermal radiation emitted by a gas layer under this angle.

On the other hand, the propagation length through a thin layer of depth $d z$ is increasing with $d l=d z / \cos \beta$, so that the $\beta$-dependence for both terms $I_{\nu, \Omega}$ and $B_{\nu, \Omega}$ disappears.
Therefore, analogous to (54) and (55) integration of (88) over $\Omega$ gives for the spectral intensity, propagating in $z$-direction:

$$
\begin{aligned}
& \frac{\int d I_{\nu, \Omega}(z) \cos \beta d \Omega}{d z} \\
& =\bar{\alpha}_{n m}(\nu) \int_{0}^{2 \pi} \int_{0}^{\pi / 2}\left(-I_{\nu, \Omega}\left(z, T_{E}\right)+B_{\nu, \Omega}\left(T_{G}(z)\right)\right) \\
& \times \sin \beta d \beta d \varphi,
\end{aligned}
$$

or with the definition for $I_{v}=\pi \cdot I_{\nu, \Omega}$ and analogous $B_{v}=$ $\pi \cdot B_{\nu, \Omega}$ :

$$
\frac{d I_{\nu}(z)}{d z}=2 \bar{\alpha}_{n m}(\nu)\left(-I_{\nu}\left(z, T_{E}\right)+B_{\nu}\left(T_{G}(z)\right)\right) .
$$

Equation (92) is a 1st-order differential equation with the general solution:

$$
\begin{aligned}
I_{\nu}(z)= & e^{-2 \int_{0}^{z} \bar{\alpha}_{n m}\left(v, z^{\prime \prime}\right) d z^{\prime \prime}} \\
& \times\left[2 \int_{0}^{z} \bar{\alpha}_{n m}\left(\nu, z^{\prime}\right) B_{\nu}\left(T_{G}\left(z^{\prime}\right)\right)\right. \\
& \left.\quad \times e^{2 \int_{0}^{z^{\prime}} \bar{\alpha}_{n m}\left(v, z^{\prime \prime}\right) d z^{\prime \prime}} d z^{\prime}+I_{\nu}(0)\right]
\end{aligned}
$$

or after some transformation:

$$
\begin{aligned}
I_{\nu}(z)= & I_{\nu}(0) e^{-2 \int_{0}^{z} \bar{\alpha}_{n m}\left(v, z^{\prime \prime}\right) d z^{\prime \prime}} \\
& +2 \int_{0}^{z} \bar{\alpha}_{n m}\left(\nu, z^{\prime}\right) B_{\nu}\left(T_{G}\left(z^{\prime}\right)\right) e^{-2 \int_{z^{\prime}}^{z} \bar{\alpha}_{n m}\left(v, z^{\prime \prime}\right) d z^{\prime \prime}} d z^{\prime} .
\end{aligned}
$$

Usually the density of the gas, the total pressure and the temperature are changing over the propagation path of the radiation. Therefore, (94) has to be solved stepwise for thin layers of thickness $\Delta z$, over which, $\bar{\alpha}_{n m}$ and $B_{v}$ can be assumed to be constant (see Figure 12). With the running index $i$ for different layers, then (94) can be simplified as follows:

$$
I_{\nu}^{i}(\Delta z)=I_{\nu}^{i-1} e^{-2 \bar{\alpha}_{n m}^{i}(v) \Delta z}+B_{\nu}^{i}\left(T_{G}^{i}\right) \cdot\left(1-e^{-2 \bar{\alpha}_{n m}^{i}(v) \Delta z}\right) .
$$

The intensity in the $i$ th layer is calculated from the previous intensity $I_{\nu}^{i-1}$ of the $(i-1)$ th layer with the values $\bar{\alpha}_{n m}^{i}(v)$ and $B_{\nu}^{i}\left(T^{i}\right)$ of the $i$ th layer. In this way, the propagation over the full atmosphere is stepwise calculated. The first term in (95) describes the transmission of the incident spectral intensity over the layer thickness, while the second term represents the self-absorption of the thermal background radiation into forward direction and is identical with the spontaneous emission of the layer into one hemisphere.

4.3. Schwarzschild Equation in a Thin Gas. Equation (88) was derived under the assumption of a local thermodynamic equilibrium in the gas, which is generally the case at higher gas pressures and therefore higher collision rates of the 


\begin{tabular}{|llll|}
\hline$I_{\nu}^{i+1}$ & $\uparrow$ & $T_{G}^{i+1}, \bar{\alpha}_{n m}^{i+1}(\nu), B_{\nu}^{i+1}\left(T_{G}^{i+1}\right)$ & \\
\hline$I_{\nu}^{i}$ & $\uparrow$ & $T_{G}^{i}, \bar{\alpha}_{n m}^{i}(\nu), B_{\nu}^{i}\left(T_{G}^{i}\right)$ & \\
\hline$I_{\nu}^{i-1}$ & $\uparrow$ & $T_{G}^{i-1}, \bar{\alpha}_{n m}^{i-1}(\nu), B_{\nu}^{i-1}\left(T_{G}^{i-1}\right)$ & $\Delta z$ \\
\hline
\end{tabular}

FIGURE 12: Stepwise calculation of the spectral intensity by the radiation transfer equation.

molecules. At low pressures, however, the population of the molecular states can also significantly be determined by induced transitions caused by the incident radiation (see Section 3.4), and then it gives more sense to consider the monochromatic or spectral radiation equilibrium, which means that the gas re-emits all monochromatic radiation it has absorbed.

Since this absorption is caused by the total spectral radiation incident within the solid angle $\Omega=2 \pi, I_{\nu, \Omega}$ has to be integrated over $\Omega$. Then the absorbed spectral intensity over the interval $d r$ is found from (51) together with (53). This must be equal to the spectral power density emitted by the gas as spontaneous emission into the full solid angle $4 \pi$. Thus, we obtain

$$
\begin{aligned}
\bar{\alpha}_{n m}(\nu) & \int_{\Omega} I_{\nu, \Omega}\left(r, T_{E}\right) \cos \beta d \Omega \\
= & \bar{\alpha}_{n m}(\nu) I_{\nu}\left(r, T_{E}\right) \equiv h \nu_{m n} A_{m n} N_{m} g(\nu) .
\end{aligned}
$$

Only $1 / 4 \pi$ of (96) contributes to the spectral radiance, and (86) changes to

$$
\frac{d I_{\nu, \Omega}}{d r}=-\bar{\alpha}_{n m}(\nu) I_{\nu, \Omega}\left(r, T_{E}\right)+\frac{1}{4 \pi} \bar{\alpha}_{n m}(\nu) I_{\nu}\left(r, T_{E}\right) .
$$

With $I_{\nu}=\pi I_{\nu, \Omega}$, (97) becomes

$$
\frac{d I_{\nu, \Omega}}{d r}=-\frac{3}{4} \bar{\alpha}_{n m}(\nu) I_{v, \Omega}\left(r, T_{E}\right) .
$$

This differential equation for the spectral radiance in thin gases was derived, only assuming the absorption and subsequent emission due to the external radiation and in this respect orientated at the standard considerations as found in the literature (see, e.g., [12]). A more rigorous derivation of this equation, however, also has to take into account the emission originating from the thermal background radiation of the molecules, as long as their temperature and thus their kinetic energy are not zero. This is considered in the next subsection.

Extended Schwarzschild Equation in a Thin Gas. We start from (86), which already describes quite generally the propagation of radiation under conditions when a sample not only absorbs but also emits on the same frequencies as the incident radiation. Again requesting radiation equilibrium, the total balance of absorption and emission processes is given by the rate equations (76), which under stationary conditions results in (see (81))

$$
\left(W_{n m}^{G}+W_{n m}^{E}+C_{n m}\right) N_{n}=\left(W_{m n}^{G}+W_{m n}^{E}+A_{m n}+C_{m n}\right) N_{m} .
$$

With (34) and (59) we find:

$$
\begin{aligned}
\left(\frac{g_{m}}{g_{n}}\right. & \left.\frac{c / n}{h \nu_{m n}} \frac{A_{m n} \lambda_{m n}^{2}}{8 \pi n^{2}}\left(u_{v}+\frac{n}{c} I_{\nu}\right)+C_{n m}\right) N_{n} \\
& =\left(\frac{c / n}{h \nu_{m n}} \frac{A_{m n} \lambda_{m n}^{2}}{8 \pi n^{2}}\left(u_{v}+\frac{n}{c} I_{\nu}\right)+A_{m n}+C_{m n}\right) N_{m} .
\end{aligned}
$$

Some rearrangement of (100) and multiplication with $h v_{m n}$ gives

$$
\begin{gathered}
\frac{A_{m n} \lambda_{m n}^{2}}{8 \pi n^{2}}\left(\frac{g_{m}}{g_{n}} N_{n}-N_{m}\right)\left(\frac{c}{n} u_{v}+I_{\nu}\right) \\
+h v_{m n}\left(C_{n m} N_{n}-C_{m n} N_{m}\right) \\
=h v_{m n} A_{m n} N_{m} .
\end{gathered}
$$

At local thermodynamic equilibrium, we know that due to (36), the term $C_{n m} N_{n}-C_{m n} N_{m}$ will be zero, and trivially it is also zero in the absence of any collisions $\left(C_{m n}=C_{n m}=0\right)$. Thus, we can expect that in a thin gas with only few collisions even under radiation equilibrium, this term is quite small and therefore can be neglected. The general case, including this term, will be discussed in the next paragraph. So, with (33) and dividing (101) by $4 \pi$ as well as using the relations $(c / 4 \pi n)$. $u_{v}=B_{v, \Omega}$ and $I_{v}=\pi I_{v, \Omega}$, we get

$$
\begin{aligned}
\frac{1}{4 \pi} \bar{\alpha}_{n m}\left(\frac{c}{n} u_{\nu}+I_{\nu}\right) & =\bar{\alpha}_{n m}\left(B_{\gamma, \Omega}+\frac{1}{4} I_{\nu, \Omega}\right) \\
& =\frac{h \nu_{m n}}{4 \pi} A_{m n} N_{m} g(\nu) .
\end{aligned}
$$

Inserting (102) in (86) then gives the extended Schwarzschild equation in thin gases:

$$
\frac{d I_{\nu, \Omega}(r)}{d r}=-\frac{3}{4} \bar{\alpha}_{n m}(\nu) I_{\nu, \Omega}\left(r, T_{E}\right)+\bar{\alpha}_{n m}(\nu) B_{\nu, \Omega}\left(T_{G}(r)\right) .
$$

4.4. Generalized Schwarzschild Equation. The objective is to find an expression which describes the radiation transfer for both limiting cases, at high collision rates in a dense gas, as given by (88), as well as at negligible collisions in a thin gas, represented by (103). For similar considerations, however, different results; see, for example, [12].

We start from (81) or with the abbreviations of (83) from (84). These equations again contain the balance of all up and down transitions. Elementary transformation yields

$$
\left(U_{v}+\kappa+\varepsilon \cdot e^{h v_{m n} /\left(k T_{G}\right)}\right) N_{m}=\frac{g_{m}}{g_{n}}\left(U_{v}+\varepsilon\right) N_{n},
$$


and some further rearrangements give (see on the left side the expansion by $+1-1$ )

$$
\begin{gathered}
\left(\kappa+\varepsilon\left(e^{h v_{m n} /\left(k T_{G}\right)}+1-1\right)\right) N_{m} \\
=\left(\frac{g_{m}}{g_{n}} N_{n}-N_{m}\right) U_{v}+\frac{g_{m}}{g_{n}} \varepsilon N_{n}, \\
\left(\kappa+\varepsilon\left(e^{h v_{m n} /\left(k T_{G}\right)}-1\right)\right) N_{m}=\left(\frac{g_{m}}{g_{n}} N_{n}-N_{m}\right)\left(U_{v}+\varepsilon\right), \\
\left.N_{m}=\frac{N_{n}\left(g_{m} / g_{n}-N_{m} / N_{n}\right)\left(U_{v}+\varepsilon\right)}{\kappa+\varepsilon\left(e^{h v_{m n}} /\left(k T_{G}\right)\right.}-1\right)
\end{gathered}
$$

With the identity (see (33) and (83))

$$
\bar{\alpha}_{n m}=\frac{h \nu_{m n}}{c / n} \frac{A_{m n}}{\kappa} N_{n}\left(\frac{g_{m}}{g_{n}}-\frac{N_{m}}{N_{n}}\right) g(\nu)
$$

and multiplying (105) with $\left(h v_{m n} / 4 \pi\right) \cdot A_{m n} g(\nu)$, we obtain

$$
\begin{aligned}
\frac{h \nu_{m n}}{4 \pi} A_{m n} N_{m} g(\nu) & =\frac{c / n}{4 \pi} \frac{\bar{\alpha}_{n m}\left(U_{v}+\varepsilon\right)}{1+(\varepsilon / \kappa)\left(e^{h \nu_{m n} /\left(k T_{G}\right)}-1\right)} \\
& =\bar{\alpha}_{n m} \frac{B_{\nu, \Omega}+(1 / 4) I_{\nu, \Omega}+((c / n) / 4 \pi) \varepsilon}{1+(\varepsilon / \kappa)\left(e^{h \nu_{m n} /\left(k T_{G}\right)}-1\right)} .
\end{aligned}
$$

Defining a relative material parameter

$$
\begin{aligned}
\eta & =\frac{c / n}{4 \pi} \frac{\varepsilon}{B_{v, \Omega}}=\frac{c / n}{4 \pi} \frac{C_{n m}}{B_{n m}} \frac{c^{2}}{2 h \nu_{m n}^{3} n^{2}}\left(e^{h \nu_{m n} /\left(k T_{G}\right)}-1\right) \\
& =\frac{\varepsilon}{\kappa}\left(e^{h \nu_{m n} /\left(k T_{G}\right)}-1\right)
\end{aligned}
$$

and inserting (107) and (108) into (86), we get

$$
\begin{aligned}
\frac{d I_{\nu, \Omega}(r)}{d r}= & -\bar{\alpha}_{n m}(\nu) I_{\nu, \Omega}\left(r, T_{E}\right) \\
& +\bar{\alpha}_{n m} \frac{B_{\nu, \Omega}+(1 / 4) I_{\nu, \Omega}+\eta B_{\nu, \Omega}}{1+\eta}, \\
\frac{d I_{\nu, \Omega}(r)}{d r}= & -\bar{\alpha}_{n m}(\nu)\left(1-\frac{1 / 4}{1+\eta}\right) I_{\nu, \Omega}\left(r, T_{E}\right) \\
& +\bar{\alpha}_{n m}(\nu) B_{\nu, \Omega}\left(T_{G}(r)\right) .
\end{aligned}
$$

With the abbreviation

$$
\chi=1-\frac{1 / 4}{1+\eta}=\frac{3+4 \eta}{4+4 \eta}
$$

where $\chi$ assumes values from $3 / 4 \leq \chi \leq 1$ for $0 \leq \eta \leq \infty$, (109) can be represented in the more compact form

$$
\frac{d I_{\nu, \Omega}(r)}{d r}=\bar{\alpha}_{n m}(\nu, r)\left(-\chi \cdot I_{\nu, \Omega}\left(r, T_{E}\right)+B_{\nu, \Omega}\left(T_{G}(r)\right)\right) .
$$

This is the generalized Schwarzschild equation, which is valid at low as well as at high gas densities and where the transition from one to the other limiting case is controlled by the density dependent parameter $\eta$. According to (108) and (83) together with (36), $\eta$ may be expressed as

$$
\begin{aligned}
\eta & =\frac{\varepsilon}{\kappa}\left(e^{h v_{m n} /\left(k T_{G}\right)}-1\right)=\frac{g_{n}}{g_{m}} \frac{C_{n m}}{A_{m n}}\left(e^{h v_{m n} /\left(k T_{G}\right)}-1\right) \\
& =\frac{C_{m n}}{A_{m n}} \frac{\left(e^{h v_{m n} /\left(k T_{G}\right)}-1\right)}{e^{h v_{m n} /\left(k T_{G}\right)}} \approx \frac{C_{m n}}{A_{m n}} .
\end{aligned}
$$

Therefore, any variations of $\eta$ and thereby also of $\chi$ originate from the pressure dependent collision transfer rate $C_{m n}$, which is changing with the altitude above the earth's surface, so that all these parameters become a function of $z$. Without any collisions, $\eta$ gets zero, and thus $\chi=3 / 4$. Then (111) is identical with (103). On the other hand, at high gas densities and high collision rates with $\eta \gg 1$ and $\chi \rightarrow 1$, (111) converges towards (88).

4.5. Generalized Radiation Transfer Equation for the Spectral Intensity. In order to derive the basic radiation transfer equation (RTE) for the spectral intensity expanding in $z$ direction, analogous to (91), we have to integrate (111) over the solid angle $\Omega=2 \pi$ :

$$
\begin{gathered}
\frac{\int d I_{\nu, \Omega}(z) \cos \beta d \Omega}{d z} \\
=\bar{\alpha}_{n m}(\nu, z) \int_{0}^{2 \pi} \int_{0}^{\pi / 2}\left(-\chi(z) I_{\nu, \Omega}\left(z, T_{E}\right)+B_{\nu, \Omega}\left(T_{G}(z)\right)\right) \\
\times \sin \beta d \beta d \varphi
\end{gathered}
$$

or with the definitions of $I_{\nu}$ and $B_{\gamma}$ :

$$
\frac{d I_{\nu}(z)}{d z}=2 \bar{\alpha}_{n m}(\nu, z)\left(-\chi(z) I_{\nu}\left(z, T_{E}\right)+B_{\nu}\left(T_{G}(z)\right)\right) .
$$

This is the generalized RTE for the spectral intensity originating from a Planckian radiator of temperature $T_{E}$ and propagating through an absorbing gas layer of temperature $T_{G}$.

Similar to (92)-(94), the solution of (114) is found to be $I_{v}(z)$

$$
\begin{aligned}
= & I_{\nu}(0) e^{-2 \int_{0}^{z} \chi\left(z^{\prime \prime}\right) \bar{\alpha}_{n m}\left(v, z^{\prime \prime}\right) d z^{\prime \prime}} \\
& +2 \int_{0}^{z} \bar{\alpha}_{n m}\left(\nu, z^{\prime}\right) B_{\nu}\left(T_{G}\left(z^{\prime}\right)\right) e^{-2 \int_{z^{\prime}}^{z} \chi\left(z^{\prime \prime}\right) \bar{\alpha}_{n m}\left(v, z^{\prime \prime}\right) d z^{\prime \prime}} d z^{\prime},
\end{aligned}
$$

and a stepwise numerical integration can be obtained by

$$
I_{\nu}^{i}(\Delta z)=I_{\nu}^{i-1} e^{-2 \chi^{i} \bar{\alpha}_{n m}^{i}(\nu) \Delta z}+\frac{1}{\chi^{i}} B_{\nu}^{i}\left(T_{G}^{i}\right) \cdot\left(1-e^{-2 \chi^{i} \bar{\alpha}_{n m}^{i}(\nu) \Delta z}\right) .
$$


It should be recalled that under conditions of stronger absorption, and longer path lengths radiation emerging from the individual layers may deviate from the assumed Lambertian radiation law (see Section 3.2). This can result in a slightly too strong absorption caused by those rays propagating under larger angles to the layer normal. However, since these rays only provide relatively small contributions to the total absorption, this only causes relatively small deviations.

Another effect may result from deviations of the rays from geometric optics, caused by inhomogeneities and scattering processes in the atmosphere. Therefore, altogether it seems reasonable to apply a slightly smaller effective absorption coefficient $2 \chi^{i} \bar{\alpha}_{n m}^{i}$ in (116). The factor of two had its origin in an effective average path length through a layer of $2 \Delta z$, corresponding to an average propagation direction $\beta=60^{\circ}$. Therefore, assuming a slightly smaller average angle of $\beta \approx$ $50^{\circ}$ might approximate more realistically the conditions in the atmosphere.

\section{Simulation of Radiation Transfer in the Atmosphere}

Infrared active gases in the atmosphere have a significant influence on the energy and radiation balance of the earthatmosphere system, as this was already discussed in some detail in Section 3.3. They absorb short wavelength radiation from the sun and long wavelength radiation from the earth. This absorbed energy as well as thermal energy (after conversion to internal energy) is again reradiated uniformly into all directions. This emission is the origin of the upand downwelling radiation of the atmosphere itself, which together with any terrestrial or cloud radiation determines the total radiation observed in up- or downward direction.

The most important infrared active gases are water vapour, $\mathrm{CO}_{2}, \mathrm{CH}_{4}$, and $\mathrm{O}_{3}$, which have stronger absorption bands in the infrared, distributed over a wide spectral range from about 3 to $200 \mu \mathrm{m}$ or 50 to $3330 \mathrm{~cm}^{-1}$.

Particularly, $\mathrm{CO}_{2}$ is believed to be the main culprit of anthropogenic global warming and is made responsible for the increasing temperature over the last 150 years. Therefore, it is worthwhile to look more carefully how this gas influences the absorption and radiation balance in the atmosphere. At the same time, this is an instructive example for the useful applicability of the theoretical background presented in this paper.

5.1. Absorption and Emission Spectrum of $\mathrm{CO}_{2}$. The strongest absorption band of this molecule is located around $4.2 \mu \mathrm{m}$ or $2350 \mathrm{~cm}^{-1}$. But for the absorption-emission balance in the atmosphere, even more important is the ten times weaker absorption band at $15 \mu \mathrm{m}\left(670 \mathrm{~cm}^{-1}\right)$, which almost coincides with the spectral maximum of the terrestrial radiation (see Figure 6), and therefore primarily determines the interaction of $\mathrm{CO}_{2}$ molecules with the radiation.

Figure 13 shows the respective absorption spectrum for $380 \mathrm{ppm}$ of $\mathrm{CO}_{2}$ in dry air for different propagation lengths at a pressure of $1013 \mathrm{hPa}$ and a temperature of $288 \mathrm{~K}$. The red lines are markers indicating the center and strength
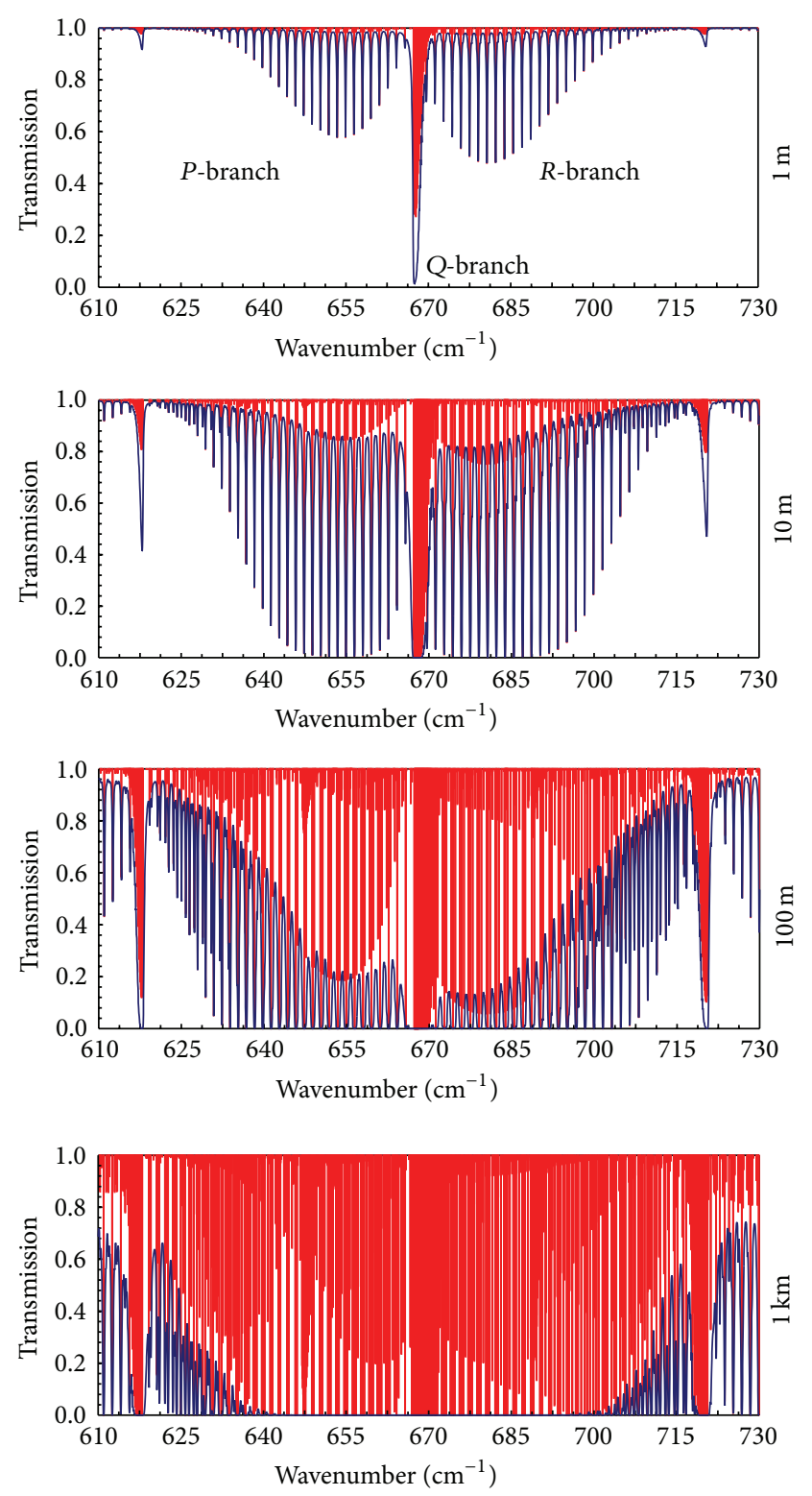

FIGURE 13: Absorption spectra for $380 \mathrm{ppm}$ of $\mathrm{CO}_{2}$ in dry air at different propagation lengths (air pressure: $1013 \mathrm{hPa}$, temperature: $288 \mathrm{~K})$.

of an individual transition, while the blue curve is the superposition of all line profiles and represents the degree of transmission-or inversely the degree of absorption-of incident radiation at these wavenumbers. On the Q-branch (band center), radiation is almost completely absorbed after a length of only $1 \mathrm{~m}$, and with increasing propagation distance, even the weaker lines of the $P$ - and $R$-branches reflect totally saturated absorption. At $1 \mathrm{~km}$ or more, only the far wings of the band structure still show some rest transmission.

On the other hand, from Sections 3 and 4, we know that an absorbing gas with a temperature comparable to that of an external radiation source-here the earth's surface-will act as own radiator and modify the incident radiation noticeably. Figure 14 displays a simulation for the upwelling radiation 


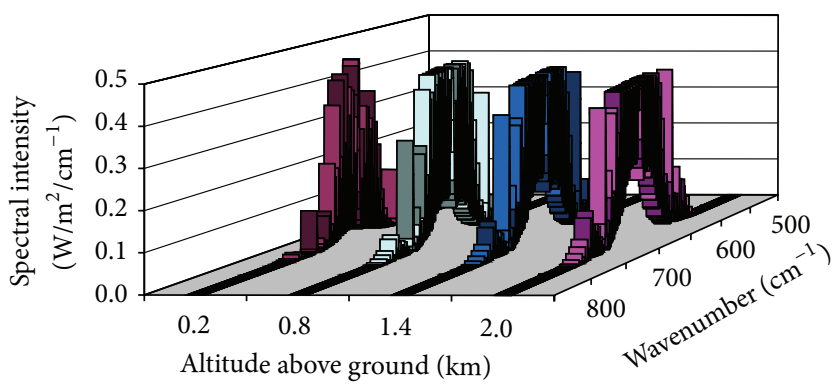

FIGURE 14: Spectral intensity radiated by $3.80 \mathrm{ppm}$ of $\mathrm{CO}_{2}$ in dry air at different altitudes (ground pressure: $1013 \mathrm{hPa}$, ground temperature of the gas: $288 \mathrm{~K})$.

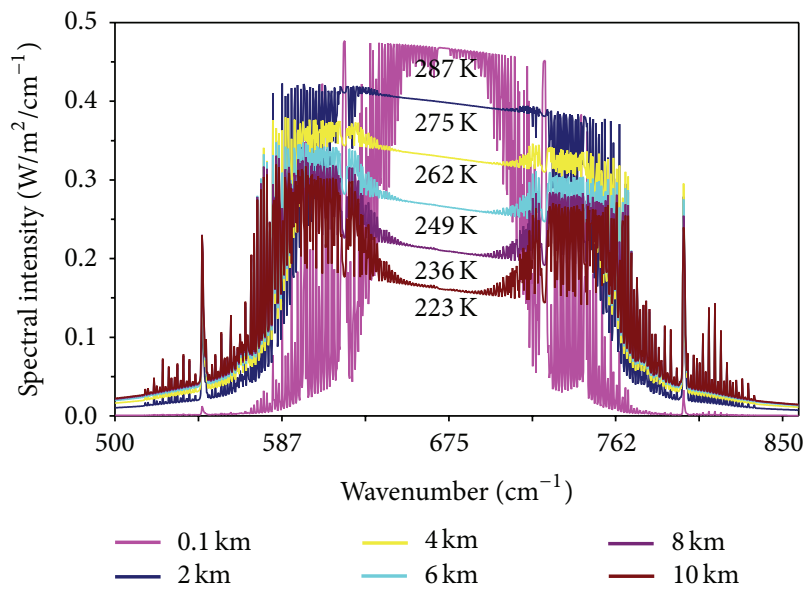

FIGURE 15: Spectral intensity emitted by $380 \mathrm{ppm}$ of $\mathrm{CO}_{2}$ in dry air at different altitudes (ground pressure: $1013 \mathrm{hPa}$, ground temperature of the gas: $288 \mathrm{~K}$ ).

emitted by only $3.8 \mathrm{ppm} \mathrm{CO}_{2}$ in dry air (1/100th of the regular concentration) at different altitudes above ground.

This calculation is based on (116) and uses for the vertical air pressure and temperature profile the US Standard Atmosphere [17]. On the band center, the radiation already rapidly attains a maximum spectral intensity of $0.47 \mathrm{~W} /\left(\mathrm{m}^{2} \cdot \mathrm{cm}^{-1}\right)$, and with increasing altitude a broader plateau is gradually developing, which at higher altitudes again slightly decreases.

This can be observed more pronounced in Figure 15 for a series of calculations up to $10 \mathrm{~km}$ altitude with a concentration of $380 \mathrm{ppm}$ of $\mathrm{CO}_{2}$ in dry air.

The broad plateaus essentially reflect the actual temperature of the gas at the specific altitudes. The higher shoulders of the curves from $4-10 \mathrm{~km}$ are an indication that with reducing pressure and thus decreasing linewidths the wings of the absorption band become more transparent, and also radiation from lower, therefore warmer, and more intensively radiating layers can contribute to the observed spectra.

All radiative transfer calculations were performed on the program-platform MolExplorer [18] as line-by-line calculations and rely on the actual HITRAN08-database [13]. For the numerical integration of (116), a layer thickness of $100 \mathrm{~m}$ was used, over which the pressure, density, and

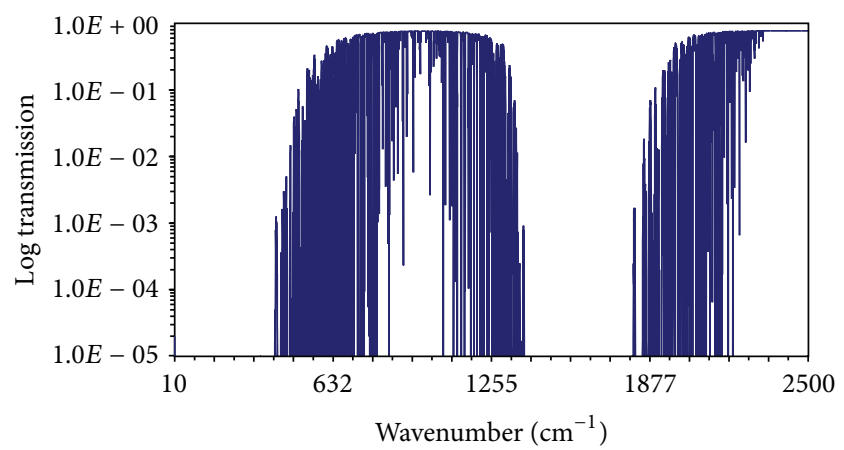

Figure 16: Transmission through wet air over $10 \mathrm{~km}$ altitude. Water vapour concentration at ground is $1.46 \%$ at $288 \mathrm{~K}$.

temperature were considered to be constant. According to (112), $\eta$ is determined by the ratio $C_{m n} / A_{m n}$. Since up to the mesopause, the collision rate $C_{m n}$ is still larger than $1 \mathrm{~s}^{-1}$ and the spontaneous emission rate on the $\mathrm{CO}_{2}$ transitions $A_{m n} \leq 1 \mathrm{~s}^{-1}, \eta$ is larger 1 and thus $\chi \approx 1$. Therefore, for this absorption band the original Schwarzschild equation (88) is still a good approximation up to an altitude of about $90 \mathrm{~km}$.

5.2. Influence of Water Vapour. The strongest impact on the radiation in the atmosphere originates from water in its different aggregate states. So, thicker cumulus or altostratus cloud layers can totally absorb and reradiate infrared radiation, or ice crystals in the tropopause may cause scattering of the radiation. But also atmospheric water vapour has a significant influence, which with respect to the scope of this paper will be considered in more detail. This strong impact results from the fact that $\mathrm{H}_{2} \mathrm{O}$ molecules possess strong absorption bands and single absorption lines which are almost continuously spread over broader regions of the infrared spectrum.

Figure 16 shows the spectral transmission through wet air on a logarithmic scale over an altitude of $10 \mathrm{~km}$. The water vapour concentration at the ground at $288 \mathrm{~K}$ is assumed to be $1.46 \%$ as an average over the three climate zones tropics, mid-latitudes, and the polar regions. It is deduced from GPS measurements [19] and decreases exponentially with altitude because of the temperature dependence of the vapour pressure (for details, see [20]).

From $10-360 \mathrm{~cm}^{-1}$ and $1400-1800 \mathrm{~cm}^{-1}$, the atmosphere is completely opaque. Only between 800 and $1100 \mathrm{~cm}^{-1}$ and above $2200 \mathrm{~cm}^{-1}$, the so-called atmospheric windows, it becomes quite transparent for radiation from the earth's surface.

But at the same time, the water molecules are strong emitters, radiating at their respective temperature, as this is represented in Figure 17. As direct comparison are also shown Planck distributions of different temperatures. Although water molecules are ideal blackbody radiators on their stronger transitions, their spectral intensity is significantly lower than the surface emission of the earth. Again this results from the fact that the spectral components observed at the tropopause in $12.5 \mathrm{~km}$ height originate from layers of different 


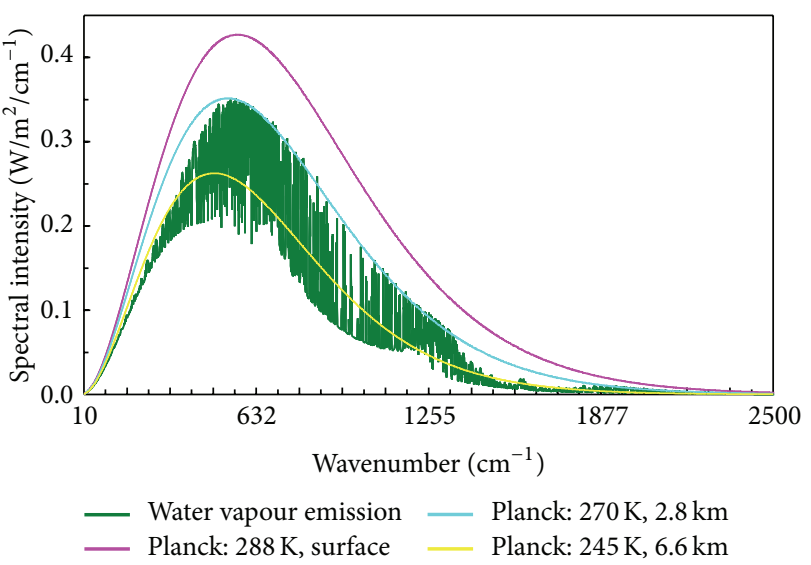

FIGURE 17: Water vapour emission at $12.5 \mathrm{~km}$ altitude. Concentration at ground is 14,615 ppm. As comparison are shown Planck curves at $288 \mathrm{~K}, 270 \mathrm{~K}$, and $245 \mathrm{~K}$.

altitude and therefore different temperature. These layers may be characterized by an optical depth $\tau_{n m}(\nu)=\bar{\alpha}_{n m}(\nu) \Delta z \approx 1$ at frequency $\nu$. Otherwise a new absorption-emission cycle would occur within the layer thickness or over the further path up to the observation altitude.

So, within spectral regions of medium or lower absorption the envelope of the emission curve can be represented quite well by a Planck distribution of $270 \mathrm{~K}$, corresponding to an altitude of $2.8 \mathrm{~km}$, while in the spectral intervals of very high opacity $\left(10-400 \mathrm{~cm}^{-1}\right.$ and $\left.1350-1800 \mathrm{~cm}^{-1}\right)$, a distribution with $245 \mathrm{~K}$ and an altitude of $6.6 \mathrm{~km}$ fits much better.

5.3. Radiation Transfer under Atmospheric Conditions. While it was the objective of the previous two subsections, to present the individual spectral properties of the two most important infrared active gases in the atmosphere and to identify their interaction with the atmospheric radiation, in this paragraph we investigate their common influence on the up- and downwelling radiation. Figure 18 shows the impact of both gases on the emitted radiation at $12.5 \mathrm{~km}$ altitude.

The blue curve represents the total radiation, emitted from the earth's surface and the atmosphere, while the contribution only from the atmosphere is depicted as green graph. Below $750 \mathrm{~cm}^{-1}$ and above $1300 \mathrm{~cm}^{-1}$ both curves are completely identical and appear as a unique line. Only over the central atmospheric window, the influence of the underlying terrestrial radiation, shown as pink graph, can be identified as increased intensity, while in the outer regions, this radiation is completely absorbed by water vapour and $\mathrm{CO}_{2}$. Similar to Figure 17, the dominant influence of the water lines over the whole spectral range can be seen, but now superimposed by the strong absorption band of $\mathrm{CO}_{2}$ around $670 \mathrm{~cm}^{-1}$. The water vapour concentration at ground again was assumed to be $14,615 \mathrm{ppm}$.

To assess the more or less strong influence of increasing $\mathrm{CO}_{2}$ concentrations in the atmosphere, as heavily discussed with respect to anthropogenic global warming, Figure 19 compares two simulations, which were performed with

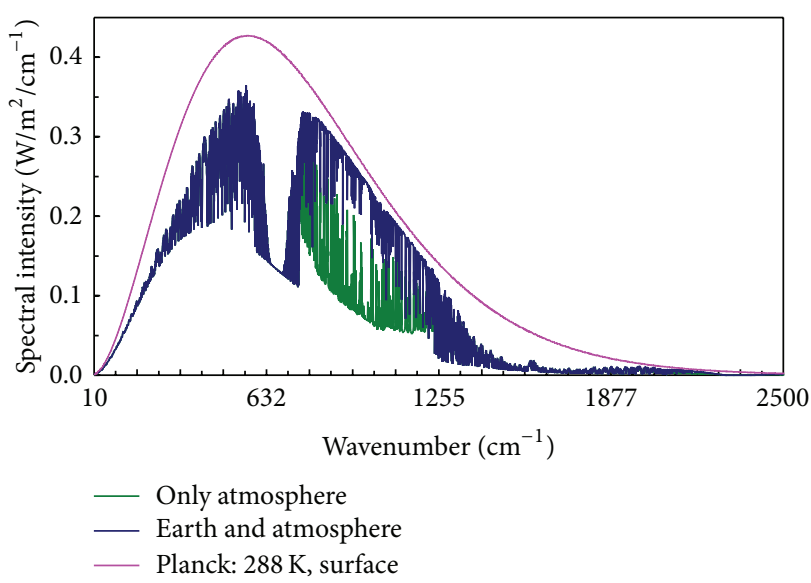

Figure 18: Total upwelling radiation in $12.5 \mathrm{~km}$ altitude (blue) and emission of only the atmosphere by water vapour and $\mathrm{CO}_{2}$ (green). Surface radiation is shown as pink line.

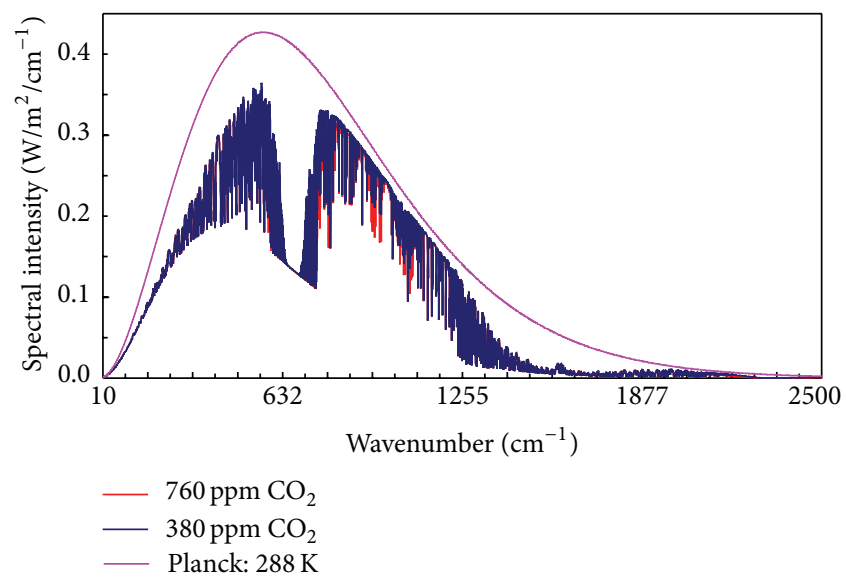

FIGURE 19: Upwelling radiation at $12.5 \mathrm{~km}$ altitude with $380 \mathrm{ppm}$ (blue line) and $760 \mathrm{ppm} \mathrm{CO}_{2}$ (red line). Water vapour concentration is $14,615 \mathrm{ppm}$ and temperature at ground $288 \mathrm{~K}$.

$380 \mathrm{ppm}$ (blue line) and $760 \mathrm{ppm}$ (red curve) of $\mathrm{CO}_{2}$ at average global conditions. Due to the strongly saturated absorption and emission on the $\mathrm{CO}_{2}$ band around $670 \mathrm{~cm}^{-1}$, the broad funnel changes neither its depth nor its width. Only the wings and the weak absorption bands at 970 and $1070 \mathrm{~cm}^{-1}$ which are all strongly superimposed by intense water lines contribute to a slightly higher absorption.

Subtraction of the integrated spectra yields an intensity difference of $5.8 \mathrm{~W} / \mathrm{m}^{2}$; so, this amount is less reradiated by the troposphere at doubled $\mathrm{CO}_{2}$ concentration. While these calculations were done under clear sky conditions, at an average cloudiness of $50 \%$ and a cloud altitude of $5 \mathrm{~km}$, this reduces to about $4.3 \mathrm{~W} / \mathrm{m}^{2}$. Under new quasiequilibrium conditions, it is found that $61 \%$ of this amount amplifies the back radiation, the other $39 \%$ go to space (see also [20]). An enhanced downward emission from the stratosphere at higher $\mathrm{CO}_{2}$ concentration $[21,22]$ is partly shielded by the cloudiness and in the radiation balance further compensated 


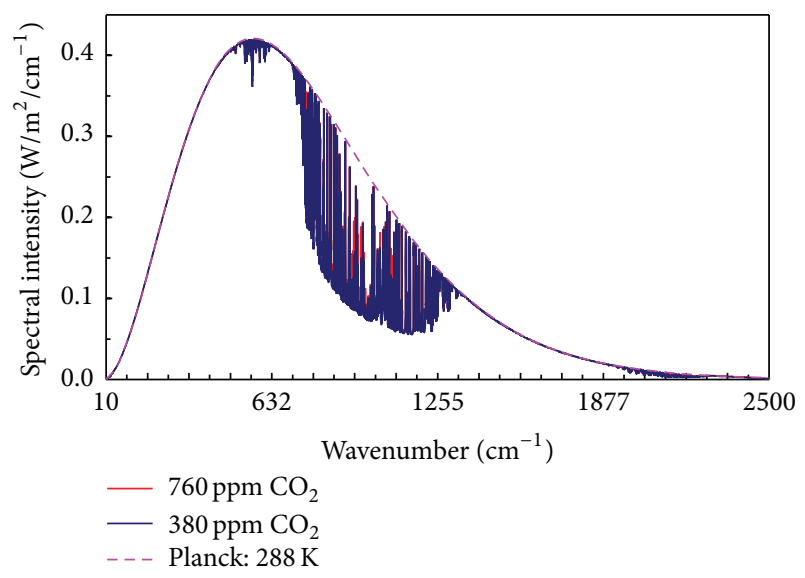

FIgURE 20: Downwelling radiation from TOA to ground with $380 \mathrm{ppm}$ (blue line) and $760 \mathrm{ppm} \mathrm{CO}_{2}$ (red line). Water vapour concentration is $14,615 \mathrm{ppm}$ and temperature at ground $288 \mathrm{~K}$.

by increased losses of the sun flux, which is absorbed by the earth's surface (shortwave radiative forcing). So, altogether about $2.6 \mathrm{~W} / \mathrm{m}^{2}$ would contribute to the radiative forcing and thus to global heating at doubled $\mathrm{CO}_{2}$ concentration.

An example for the downwelling radiation is shown in Figure 20. The calculations start at the intersection of the mesosphere to the mesopause in $86 \mathrm{~km}$ altitude (TOA) and track the emission as well as the absorption down to the earth's surface. The simulations again include water vapour in the troposphere with the ground concentration of $14,615 \mathrm{ppm}$, $\mathrm{CO}_{2}$, and $\mathrm{CH}_{4}$ as well mixed gases over the whole altitude with concentrations for $\mathrm{CO}_{2}$ of $380 \mathrm{ppm}$ (blue line) and $760 \mathrm{ppm}$ (red line), for $\mathrm{CH}_{4}$ with $1.8 \mathrm{ppm}$ and $\mathrm{O}_{3}$ spread over the stratosphere and tropopause with a maximum of $7 \mathrm{ppm}$ around $38 \mathrm{~km}$ altitude. Except the smaller contribution from $\mathrm{O}_{3}$ around $1020 \mathrm{~cm}^{-1}$, all the radiation originates from the lower troposphere and is over wide regions identical with a blackbody source of $288 \mathrm{~K}$. The integral radiation in downward direction is $312 \mathrm{~W} / \mathrm{m}^{2}$, representing $80 \%$ of a blackbody source at that temperature and corresponding to $61 \%$ of the total intensity, radiated only by the atmosphere. The difference between ordinary and doubled $\mathrm{CO}_{2}$ concentrations at clear sky is $4.4 \mathrm{~W} / \mathrm{m}^{2}$, while at average cloudiness that amount, which contributes to an additional heating of the earth, is the same as discussed above.

The calculated radiative forcing of $2.6 \mathrm{~W} / \mathrm{m}^{2}$ at doubled $\mathrm{CO}_{2}$ concentration is $1.1 \mathrm{~W} / \mathrm{m}^{2}$ smaller than that of Myhre et al. [22]. Up to some degree, this discrepancy is expected to result from different atmospheres used for the calculations. So, most of the spectroscopic computations in the literature are based on the US Standard 1976 Atmosphere. However, the corresponding water vapour concentration of this data basis is only half of that found in the average global atmosphere (AGA) and also only half of that derived from actual GPS measurements [19], as used in our calculations. Further deviations might result from different concepts, for example, not assuming thermal equilibrium or calculating with different effective path lengths of the radiation through the atmosphere.

To demonstrate the reliability and validity of the presented simulations, we compare our calculations with two measurements, which were recorded under quite different conditions. Figure 21(a) shows a measurement of the outgoing infrared emission spectrum of the cloud-free atmosphere as detected by a satellite in $20 \mathrm{~km}$ altitude looking downward over the polar ice sheet [23].

The simulation shown in Figure 21(b) was performed for a ground temperature of $266 \mathrm{~K}$, a water vapour content at the surface of $2100 \mathrm{ppm}, \mathrm{CO}_{2}$ concentration of $380 \mathrm{ppm}$ (blue graph) and $760 \mathrm{ppm}$ (red line), $\mathrm{a} \mathrm{CH}_{4}$ concentration of $1.8 \mathrm{ppm}$, and a slightly varying $\mathrm{O}_{3}$ concentration of 1-3 ppm over the tropopause.

On the other hand, Figure 22(a) gives an example for the downwelling radiance recorded by a ground station in Reno over one day with strongly varying cloud coverage [24]. Figure 22(b) shows the simulations based on (111) but superimposed by a Planckian distribution, which accounts for the emitted radiation of the clouds. This Planck radiation is defined by the cloud temperature and thus the cloud altitude, and it contributes to the spectrum with a weighting factor given by the cloud coverage.

The ground temperatures varied from 282 to $288 \mathrm{~K}$ over the day, and the water vapour concentration was assumed to be $3000 \mathrm{ppm}$, while for the other gases, the standard conditions were applied.

All characteristic features of the measured spectra are well reproduced. The smaller deviations in the shape of the $\mathrm{CO}_{2}$ funnel as well as in the form of the downward radiation at $670 \mathrm{~cm}^{-1}$ and also discrepancies in the $\mathrm{O}_{3}$ band structure around $1050 \mathrm{~cm}^{-1}$ mainly originate from a lack of knowledge of some experimental parameters. So, the resolution of the spectrometers and also the acceptance angle of the detection optics have significant influence on the shape, widths, and intensity of the measured spectra. But also inaccurate or missing atmospheric parameters like the pressure and temperature profile over the path length during the measurements, or the vapour and gas concentrations, will limit the exact reproducibility of the measured spectra.

It should be emphasized that the measurements in Figures 21 and 22 show the up- and downwelling spectral radiance, that is, radiation, which is collected within a restricted solid angle. This angle is normally determined by the receiver optic, which causes a spatial filtering and essentially detects rays, transmitting the atmosphere on a shorter way (mostly perpendicular to the earth's surface). In contrast to this, Figures 19 and 20 were calculated for the spectral intensity, which spreads out over the whole hemisphere in upward or downward direction and corresponds to an average path length about twice that of the radiance. Therefore, due to the shorter effective interaction length particularly the wings of the spectral bands and lines in Figures 21 and 22 appear less broadened and saturated. Additionally, at the lower temperatures and dryer air, the water lines are less dominant, so that under clear sky conditions the atmosphere gets much more transparent, particularly within the spectral window from 800 to $1200 \mathrm{~cm}^{-1}$. 


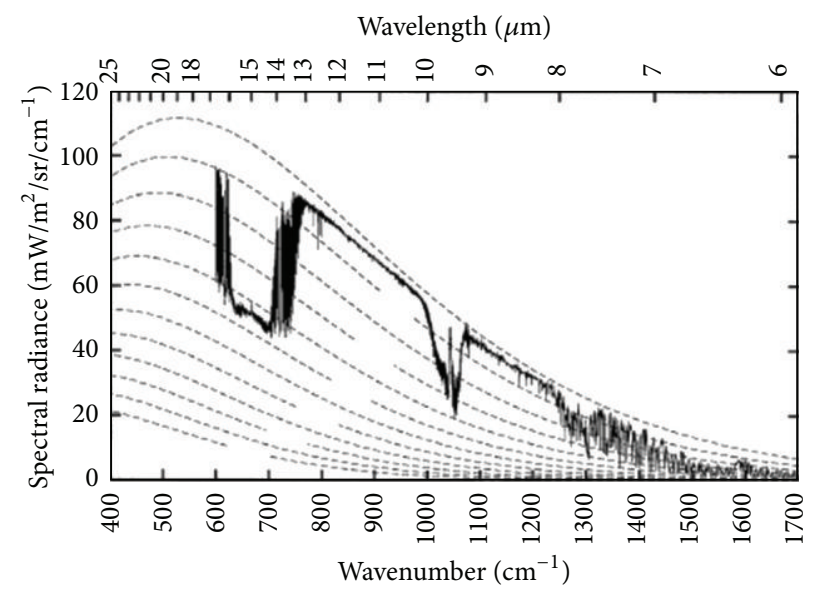

(a)

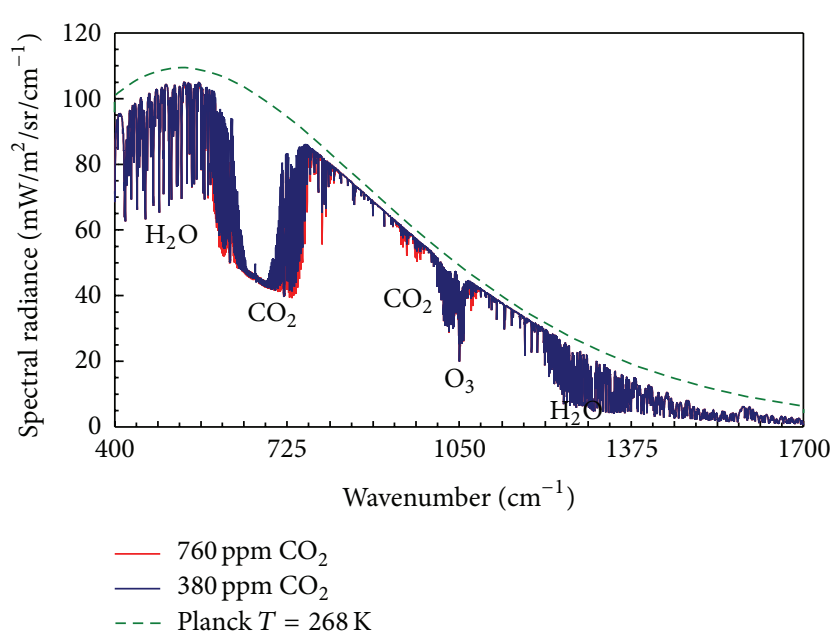

(b)

FIGURE 21: Upwelling radiance (a) measured from a satellite over the polar ice sheet [23], (b) calculated with 380 ppm (blue line) and 760 ppm $\mathrm{CO}_{2}$ (red line). Water vapour concentration is $2100 \mathrm{ppm}$ and ground temperature $268 \mathrm{~K}$.

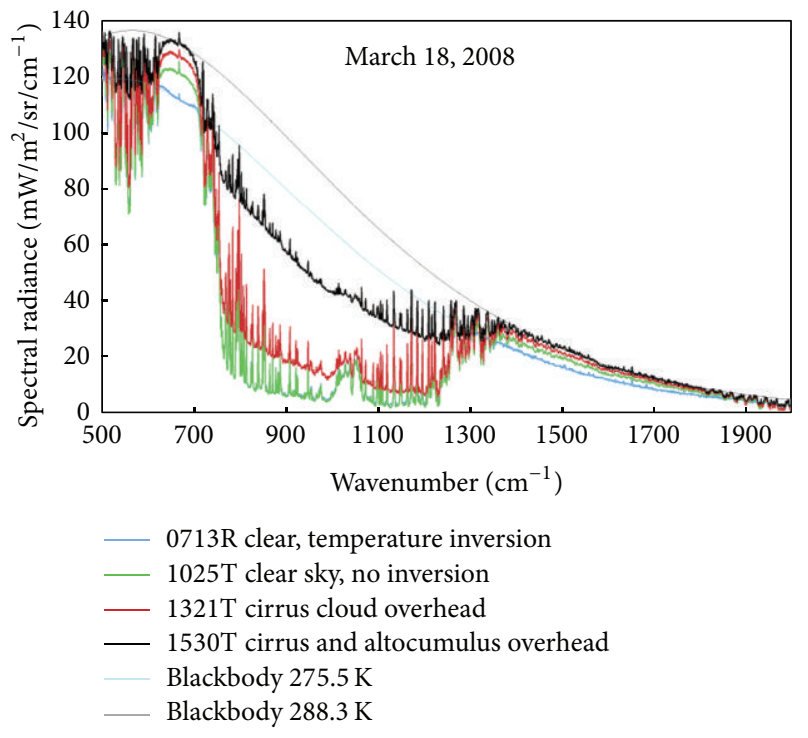

(a)

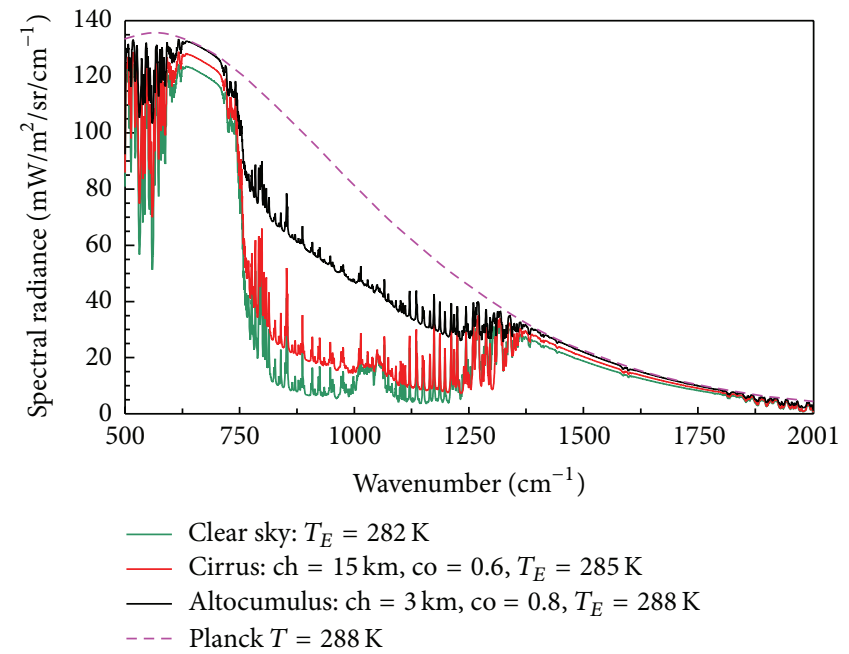

(b)

FIGURE 22: Downwelling radiance (a) measured at ground with varying cloud coverage [24], (b) calculated with water vapour concentration of $3000 \mathrm{ppm}$ and different temperatures.

These examples clearly demonstrate that the basic interaction processes of infrared molecules with thermal radiation are well understood and that the presented radiation transfer calculations are in excellent agreement with the observations, even under the quite complex conditions in the atmosphere. From this, we can also conclude that simulations of the type as shown in Figures 19-21 with different concentrations of $\mathrm{CO}_{2}$ in the atmosphere are quite reliable and give an important assessment for the impact of greenhouse gases on our climate.
5.4. Absorption of Terrestrial Radiation. The dominant influence of water vapour on the radiation and energy budget in the atmosphere can also be deduced from an analysis of pure absorption spectra. So, under conditions as discussed before with an average global water vapour content of $1.46 \%$ at the surface and a temperature of $288 \mathrm{~K}$, the overall absorption of the terrestrial radiation, only caused by water vapour, is already $77.7 \%$. Together with methane and ozone in the atmosphere, the absorption increases to $80.7 \%$. On the other hand, 


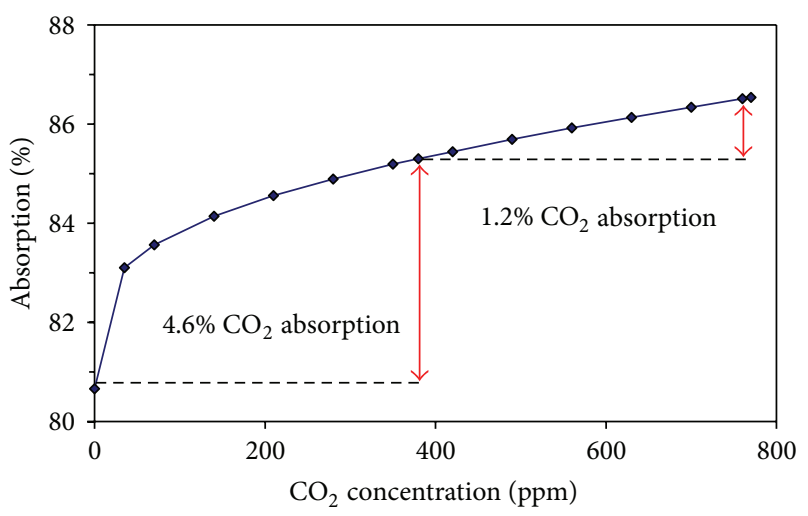

FIGURE 23: Absorption of terrestrial radiation as a function of the $\mathrm{CO}_{2}$ concentration.

$\mathrm{CO}_{2}$ alone at a concentration of $380 \mathrm{ppm}$ would contribute to $24.1 \%$, but in the presence of other gases, its influence is repelled to $4.6 \%$, which is due to the strong spectral overlap with the other components, particularly with water vapour, and the total absorption only climbs up to $85.3 \%$.

As already outlined in Sections 3.3 and 5.1, the centre of the $\mathrm{CO}_{2}$ absorption band around $670 \mathrm{~cm}^{-1}$ rapidly goes into saturation with increasing propagation length and also with increasing concentration. Only the wings and the weaker absorption bands at 970 and $1070 \mathrm{~cm}^{-1}$ still contribute to a slightly rising absorption with increasing $\mathrm{CO}_{2}$ concentration. This is shown in Figure 23, demonstrating that due to the spectral overlap and the strong saturation behaviour, a doubling of $\mathrm{CO}_{2}$ under clear sky conditions only donates additional $1.2 \%$ to the total absorption.

With cloudiness this contribution is even further pushed down to less than $1 \%$, and from this $39 \%$ will be reradiated to space. So, altogether only about $0.6 \%$, corresponding to $2.4 \mathrm{~W} / \mathrm{m}^{2}$ of the total terrestrial radiation $\left(391 \mathrm{~W} / \mathrm{m}^{2}\right.$ at $288.15 \mathrm{~K}$ ) or $0.8 \%$ with respect to the total back radiation of $312 \mathrm{~W} / \mathrm{m}^{2}$ (see Section 5.3), can contribute to an additional global heating at doubled $\mathrm{CO}_{2}$ concentration. This result is in quite good agreement with the previous calculation of the radiative forcing and demonstrates why a further increase in the $\mathrm{CO}_{2}$ concentration only gives marginal corrections in the radiation budget.

Based on the simple assumption that due to the back radiation, the natural greenhouse effect causes a global temperature increase of approximately $33 \mathrm{~K}$ and that the surface temperature linearly responds to any changes in the energy or radiation budget, an increase in this budget of less than $1 \%$ should only contribute to a temperature increase of about $0.3 \mathrm{~K}$. This is in clear contradiction to the IPCC, which issues an official climate sensitivity (temperature increase at doubled $\mathrm{CO}_{2}$ ) of $C_{S}=3.2 \mathrm{~K}$ [25]. But even a more sophisticated consideration, based on an energy balance model for the surface-atmosphere system and distinguishing between three climate zones, including the shortwave radiation budget as well as water vapour and lapse rate feedback, only gives a climate sensitivity of $0.6 \mathrm{~K}[20]$, which is still $5 \mathrm{x}$ smaller than the IPCC value.

\section{Conclusion}

The paper reviews the basic relations for the interaction of radiation with infrared active molecules and presents a new theoretical concept for the description of the radiation transfer in the atmosphere. Starting from Einstein's fundamental considerations on the quantum nature of radiation, it is shown that the thermal radiation of a gas can be well understood only in terms of the spontaneous emission of the molecules. The interaction with radiation from an external blackbody source in the presence of the background radiation and under the influence of molecular collisions is elaborately investigated. The energy and radiation balance in the atmosphere, including heat transfer processes, are described by rate equations which are solved numerically for typical conditions as found in the troposphere and stratosphere. These simulations demonstrate the continuous and dominant conversion process of heat to radiation and vice versa.

Consideration of the radiation interaction on a molecular basis allows to derive the Schwarzschild equation, describing the radiation transfer in the atmosphere. This equation is investigated under conditions of only few intermolecular collisions as found in the upper mesosphere as well as at high collision rates as observed in the troposphere. A generalized form of the radiation transfer equation is presented, which covers both limiting cases of thin and dense atmospheres and allows a continuous transition from low to high densities, controlled by a density dependent parameter. This equation is derived for the spectral radiance as well as for the spectral flux density (spectral intensity).

The radiation transfer equation is applied to simulate the up- and downwelling radiation and its interaction with the most prominent greenhouse gases water vapour, carbon dioxide, methane, and ozone in the atmosphere. The radiation in upward direction is the only possibility to render any absorbed energy to space and by this to keep the atmospheric temperature in balance with all the supplied direct and indirect energy from the sun. On the other hand, the downwelling part determines the back radiation from the atmosphere to the earth's surface, which is the source term of the so heavily discussed atmospheric greenhouse or atmospheric heating effect. While the upwelling radiation only contributes to cooling, as long as the radiation can escape to space, the downwelling part only causes heating, when it reaches the surface.

Propagation of radiation in the atmosphere is not the result of a single event. It underlies continuous absorption and reemission processes in the gas, which are determined by the mean free absorption length and strongly depend on the frequency of the radiation as well as on the gas density. Radiation also converts into molecular kinetic energy and via exciting collisions changes back to radiation. This kinetic energy of the molecules results from absorbed long- and shortwave radiation as well as from sensible and latent heat due to convection and evapotranspiration from the surface to the atmosphere.

So, any propagation of the radiation in the atmosphere can be described similar to a diffusion process of particles (photons), which are partly annihilated and again created 
while propagating through the atmosphere. This propagation in a gas is just described by the radiation transfer equation (111) for the spectral radiance and by (116) for the spectral intensity (spectral flux density), and these equations are valid independent, if an external radiation or the eigen radiation of the atmosphere in up or down direction is calculated.

An asymmetry in the up- and downwelling eigen radiation (only from atmosphere) will be found with a stronger contribution in downward direction, which is caused by the lapse rate as well as the density profile over the atmosphere with higher temperature and pressure at lower atmospheric layers and therefore a higher net downward radiation. Calculations for the radiative forcing at doubled $\mathrm{CO}_{2}$ concentration and at an average cloudiness give a $30 \%$ smaller forcing than applied by the IPCC.

Thermal radiation is electromagnetic radiation and no heat. Therefore, in the same way, as radio waves can propagate from a colder antenna to a warmer receiver, microwaves can be absorbed by a hot chicken, or $\mathrm{CO}_{2}$-laser radiation $(10.6 \mu \mathrm{m})$ can be used for welding and melting of metals up to several thousand ${ }^{\circ} \mathrm{C}$, so any back radiation from colder and higher atmospheric layers can be absorbed by the lower and warmer layers, and this back radiation can also be absorbed by a warmer surface of the earth without violating the 2 nd law of thermodynamics. As long as the surface is assumed to be a black or gray absorber, it does not filter any frequencies of the incoming radiation, in the same way as it does not reject any frequencies of the broad Planck spectrum of a thermal radiator, independent, if it has a higher or lower temperature than the earth. Radiation converts to heat after an absorption, followed by an emission in accordance with a newly adjusting thermodynamic equilibrium, which only requires that the net energy transfer is in balance.

\section{References}

[1] A. Einstein, "Quantentheorie der Strahlung," Zeitschrift für Physik, vol. 18, p. 121, 1917.

[2] M. Planck, "Über eine Verbesserung der Wienschen Spektralgleichung," Verhandlungen der Deutschen Physikalischen Gesellschaft, vol. 2, no. 5/6, pp. 519-525, 1900.

[3] L. Boltzmann, "allgemeine Sätze über Wärmegleichgewicht," Wiener Berichte, vol. 63, pp. 679-711, 1871.

[4] L. Boltzmann, "Weitere Studien über das Wärmegleichgewicht unter Gasmolekülen,” Wiener Berichte, vol. 66, pp. 275-370, 1872.

[5] A. Schuster, "Radiation through a Foggy Atmosphere," The Astrophysical Journal, vol. 21, pp. 1-22, 1905.

[6] K. Schwarzschild, "Über das Gleichgewicht in der solaren Atmosphäre-on the equilibrium of the sun's atmosphere," Nachrichten der Koniglichen Gesellschaft der Wissenschaften zu Gottingen, vol. 195, p. 41, 1906.

[7] K. Schwarzschild, "Diffusion and absorption in the sun's atmosphere," Sitz. K. Preuss. Akad. Wiss, p. 1183, 1914.

[8] G. C. Wick, “über ebene Diffusionsprobleme," Zeitschrift für Physik, vol. 121, no. 11-12, pp. 702-718, 1943.

[9] S. Chandrasekhar, "On the radiative equilibrium of a stellar atmosphere. II," Astrophysical Journal, vol. 100, article 76, 1944.

[10] R. M. Goody and Y. L. Yung, Radiation: Theoretical Basis, Oxford University Press, Oxford, UK, 1989.
[11] M. L. Salby, Physics of the Atmosphere and Climate, Cambridge University Press, Cambridge, UK, 2012.

[12] E. A. Milne, "Effect of collisions on monochromatic radiative equilibrium," The Royal Astronomical Society, vol. 88, article 493, 1928.

[13] L. S. Rothman, I. E. Gordon, A. Barbe et al., "The HITRAN 2008 molecular spectroscopic database," Journal of Quantitative Spectroscopy and Radiative Transfer, vol. 110, no. 9-10, pp. 533$572,2009$.

[14] N. Jacquinet-Husson, N. A. Scott, A. Chédin et al., "The 2003 edition of the GEISA/IASI spectroscopic database," Journal of Quantitative Spectroscopy and Radiative Transfer, vol. 95, no. 4, pp. 429-467, 2005.

[15] S. S. Penner, Molecular Spectroscopy and Gas Emissivities, Addison-Wesley, Reading, Mass, USA, 1959.

[16] C. H. Townes and A. L. Schawlow, Spectroscopy, Dover, New York, NY, USA, 1975.

[17] US Standard Atmosphere, 1976.

[18] H. Harde and J. Pfuhl, MolExplorer-A Program-Platform for the Calculation of Molecular Spectra and Radiation Transfer in the Atmosphere, Helmut-Schmidt-University Hamburg, Hamburg, Germany, 2013.

[19] S. Vey, Bestimmung und Analyse des atmosphärischen Wasserdampfgehaltes aus globalen GPS-Beobachtungen einer Dekade mit besonderem Blick auf die Antarktis [Doctoral theses], Technische Universität Dresden, Dresden, Germany, 2007.

[20] H. Harde, Was trägt $\mathrm{CO}_{2}$ wirklich zur globalen Erwärmung bei? Spektroskopische Untersuchungen und Modellrechnungen zum Einfluss von $\mathrm{H}_{2} \mathrm{O}, \mathrm{CO}_{2}, \mathrm{CH}_{4}$ und $\mathrm{O}_{3}$ auf unser Klima, Books on Demand, Norderstedt, Germany, 2011.

[21] V. Ramanathan, L. Callis, R. Cess et al., "Chemical interactions and effects of changing atmospheric trace gases," Reviews of Geophysics, vol. 25, pp. 1441-1482, 1987.

[22] G. Myhre, E. J. Highwood, K. P. Shine, and F. Stordal, "New estimates of radiative forcing due to well mixed greenhouse gases," Geophysical Research Letters, vol. 25, no. 14, pp. 27152718, 1998.

[23] Data Courtesy of D. Tobin, Space Science and Engineering Center, University of Wisconsin-Madison, Madison, Wis, USA, 1986.

[24] Graphic from Pat Arnott, University of Nevada, Reno, Nev, USA, 2008, ATMS 749.

[25] D. A. Randall, R. A. Wood, S. Bony et al., "Climate models and their evaluation, in Climate Change 2007: The Physical Science Basis," in Contribution of Working Group I to the Fourth Assessment Report of the Intergovernmental Panel on Climate Change, S. Solomon, D. Qin, M. Manning et al., Eds., Cambridge University Press, Cambridge, UK, 2007. 

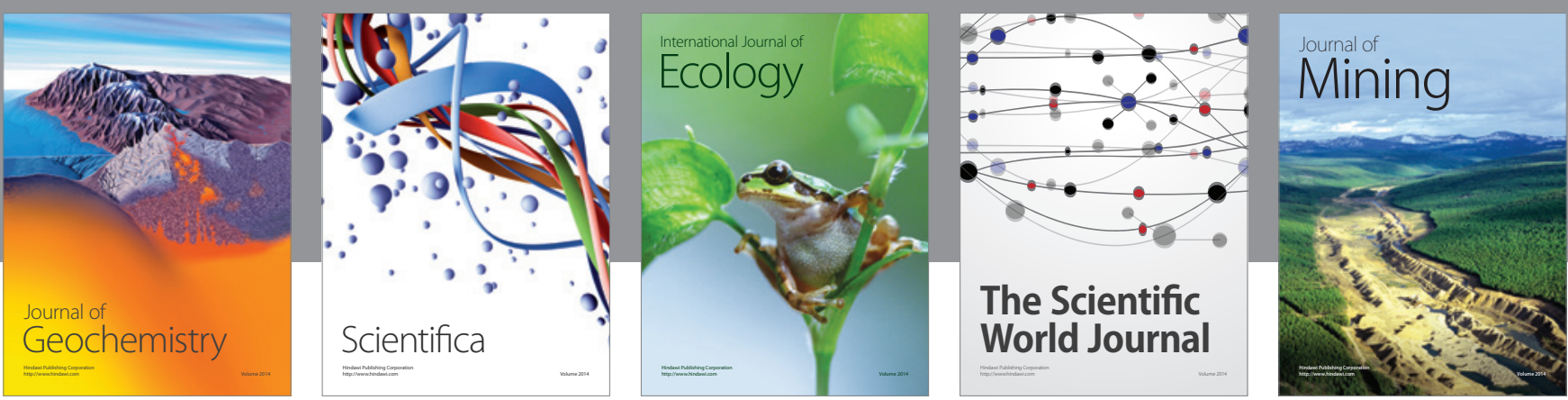

The Scientific World Journal
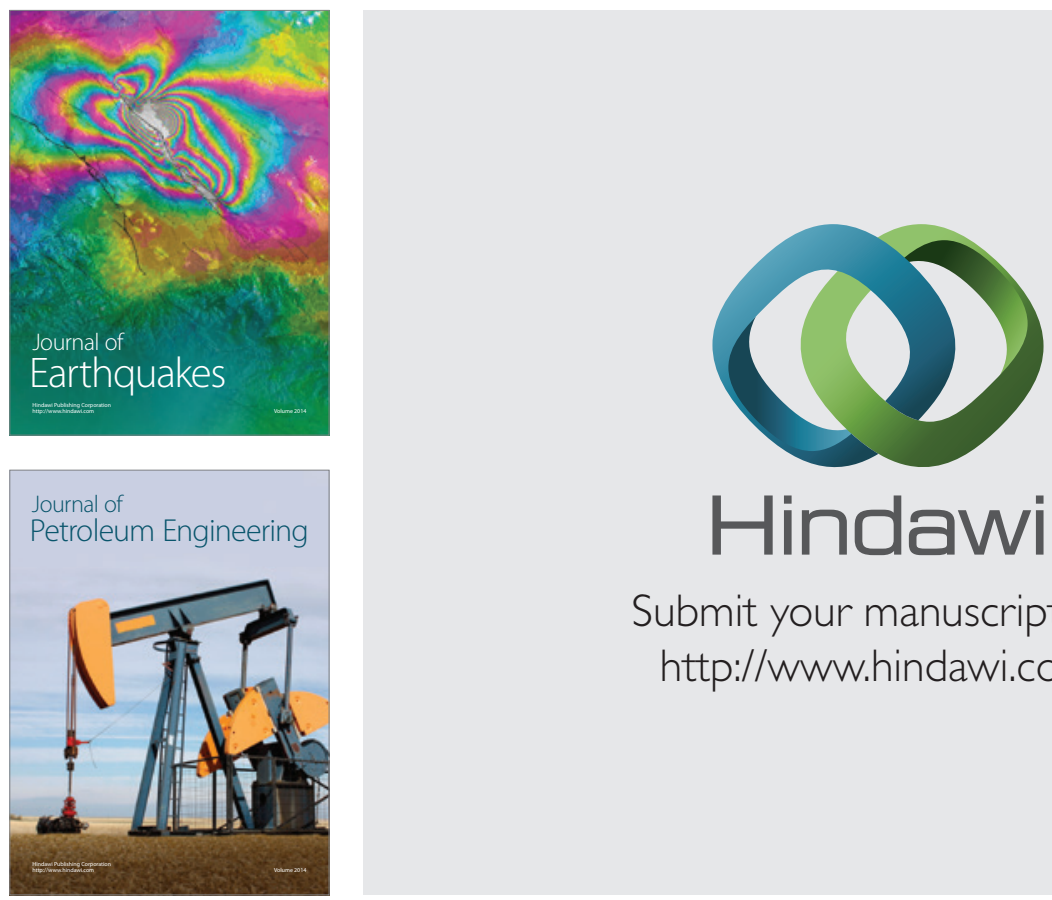

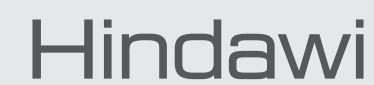

Submit your manuscripts at

http://www.hindawi.com
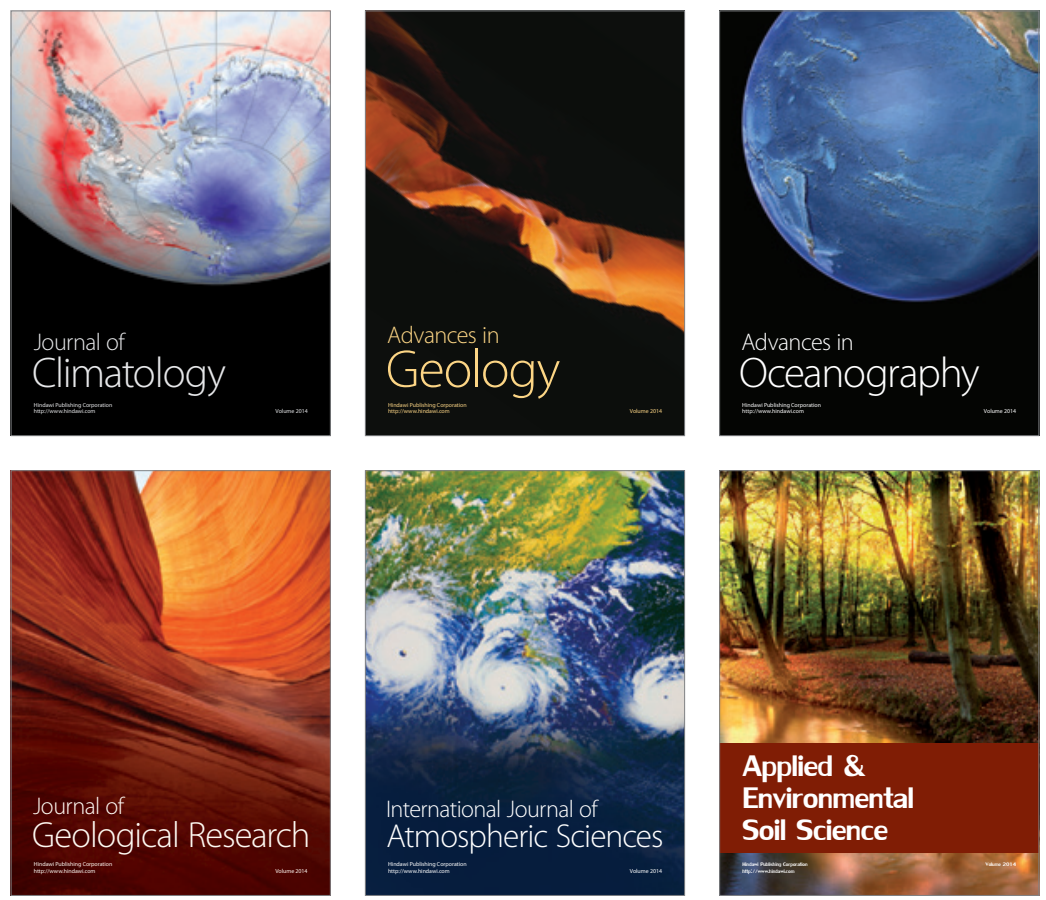
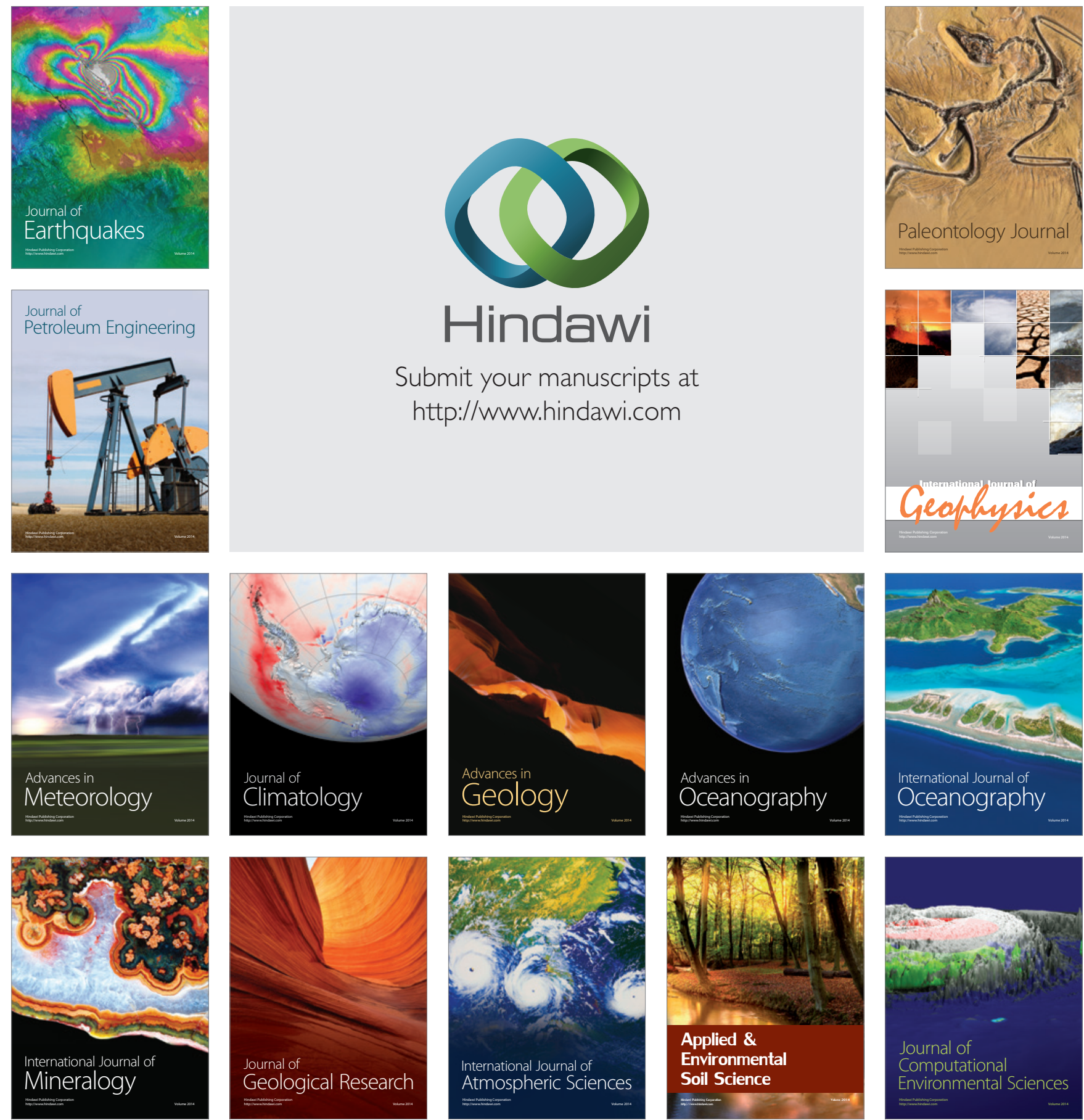